26 | 2004

Varia

\title{
Léonard de Marandé, polémiste antijanséniste
}

\section{Keisuke Misono}

\section{OpenEdition}

Journals

Édition électronique

URL : http://journals.openedition.org/ccibp/519

DOI : $10.4000 /$ ccibp. 519

ISSN : 2493-7460

\section{Éditeur}

Centre international Blaise Pascal

\section{Édition imprimée}

Date de publication : 4 mars 2005

Pagination : 7-23

ISBN : 9782845162884

ISSN : 0249-6674

Référence électronique

Keisuke Misono, «Léonard de Marandé, polémiste antijanséniste », Courrier du Centre international Blaise Pascal [En ligne], 26 | 2004, mis en ligne le 02 décembre 2015, consulté le 19 avril 2019. URL http://journals.openedition.org/ccibp/519; DOI : 10.4000/ccibp.519

Ce document a été généré automatiquement le 19 avril 2019

Centre international Blaise Pascal 


\title{
Léonard de Marandé, polémiste antijanséniste
}

\author{
Keisuke Misono
}

\section{Introduction}

1 Nous allons présenter la vie et l'œuvre de Léonard de Marandé. Sur cet adversaire de Port-Royal, on sait peu de choses. Cependant, comme nous allons le voir, les divers écrits qu'il a laissés dans sa vie, dont le nombre s'élève à plus de vingt-cinq, nous offrent souvent des sujets intéressants pour l'histoire religieuse et philosophique et notamment, sur le problème janséniste.

2 En général ses premiers écrits sur la philosophie et la théologie scolastique et ceux concernant la polémique contre Port-Royal partagent en deux la vie de cet auteur que l'on peut appeler polygraphe : jusqu'à 1649 il ne publie aucun écrit concernant la querelle janséniste, mais à partir des années 1650 il s'engage brusquement dans le mouvement anti-janséniste. La date du changement de direction de sa plume correspond à peu près à celle de sa promotion sociale, étroitement liée à la répugnance que Port-Royal éprouvait à l'égard de Marandé.

3 Dans cet exposé, après avoir examiné successivement deux parties de sa vie, nous étudierons les relations entre Marandé et Port-Royal, qui jetteront, je pense, une nouvelle lumière sur la trace de ce polémiste.

\section{Marandé vulgarisateur}

4 La famille Marandé, originaire du Berry, appartient au milieu de la noblesse de robe. Les sources importantes nous livrant des informations sur cette famille, sont d'abord des sources manuscrites conservées aux archives départementales du Cher et à la bibliothèque nationale ${ }^{1}$. Nous pouvons utiliser comme sources imprimées un ouvrage: Histoire chronologique de la grande chancellerie de France, publié par Abraham Tessereau en 
$1710^{2}$. Selon ces sources, au cours du XVII ${ }^{\mathrm{e}}$ siècle, les chefs de cette famille étaient les seigneurs de Berlières, fief situé entre Bourges et Nevers, qui faisait partie de la commune de Mornay-Berry dans le département du Cher. Mais les membres de la famille habitaient à Paris, déjà au début du siècle, et s'acquittaient de leurs fonctions publiques : Jean de Marandé, père de Léonard, habitait dans la paroisse de Saint-Landry en 1604, en travaillant comme conseiller et secrétaire de Henri IV. Son fils Charles de Marandé demeurait dans la paroisse de Saint-Paul au plus tard en 1630 ; il était maître d'hôtel ordinaire et se mettait au service de Mazarin qui l'a beaucoup apprécié3. Sur Charles de Marandé, on peut se référer à un article de Henri Courteault, historien, portant sur une de ses missions ${ }^{4}$. Le frère de Charles, Léonard de Marandé, obtiendra d'abord un emploi dans la cour des aides de Paris. A la différence de son père et son frère, il n'était pas le seigneur du fief de Berlières : en 1643 les deux frères, Léonard et Charles, ont partagé également le fief de Berlières qu'ils ont hérité de leur neveu, mais en même temps ils ont passé un contrat par lequel Charles a acquis la moitié de ce fief attribuée à Léonard. A part ce fait, nous n'avons pas de sources rattachant celui-ci au fief de Berlières.

Dans l'état actuel des recherches, la description la plus détaillée sur la vie et l'œuvre de Léonard de Marandé est l'article du Dictionnaire de théologie catholique (par Jean Carreyre, publié en 1927); il est vrai qu'on peut trouver des analyses et des mentions faites sur quelques-uns de ses livres, mais nous ne trouvons pas de travaux abordant de front cet écrivain. En fait nous ignorons les dates de sa naissance et de sa mort. Notre première rencontre avec lui date de 1624, avec la publication de son premier ouvrage intitulé Jugement des actions humaines, et nous le retrouvons, pour la dernière fois, en 1676, grâce au fait qu'il avait enrichi la traduction : Berger fidèle.

Pour aborder d'abord la première partie de sa carrière, notre examen portera sur ses écrits, ses relations et sa profession. Cette époque ne concerne pas la querelle janséniste, mais, comme nous le verrons, la future polémique évoquera certainement à Marandé et à ses adversaires la première partie de sa vie.

\section{Premiers ouvrages}

7 Avant l'année 1649 Marandé publie huit ouvrages. Dans les années 1620, la direction de ses ouvrages ne semble pas fixée. Quand il a publié en 1624 sa première œuvre Jugement des actions humaines, il se révèle être un sceptique résolu. Ce livre, dédié au cardinal de Richelieu, reflète manifestement l'influence de Montaigne dont Marandé était alors un lecteur assidu. Cet ouvrage sera traduit en anglais en 1629 ; le traducteur qui considère cet ouvrage comme le chef-d'œuvre de la philosophie morale («masterpiece of Moral philosophy »), loue l'auteur du dévoilement de la vanité des sciences humaines en tant que "another Cornelius Agrippa » et de la lutte contre les passions humaines comme « second Montaigne ${ }^{5}$. " N'entrons pas dans les détails du scepticisme de Marandé, mais nous pouvons en revanche nous référer au livre d'Alan Boase The fortunes of Montaigne (1935), qui consacre un chapitre à ce sujet. Son second ouvrage publié en 1629 est un roman intitulé : Ariades. C'est le seul livre écrit en latin parmi tous ses écrits, mais Marandé préparait en même temps l'abrégé en français, publié l'année suivante sous le titre : Abrégé de l'histoire d'Ariadés. Ne parlons pas non plus de cet ouvrage, qui ne revêt pas une grande importance. Il est à noter quand même que l'imprimeur de ces deux premiers ouvrages est la famille Cramoisy qui publiera la plupart de ses écrits polémiques. 
Dix ans après, en 1639, il a publié son troisième ouvrage intitulé Le philosophe chrétien. Cette publication est anonyme, mais les mentions de ce livre faites par lui-même dans d'autres endroits nous feront découvrir qu'il en est l'auteur 6 . Celui-ci, qui l'a rédigé pour un homme de cour, de condition élevée, ayant des doutes sur sa foi, essaie d'expliquer les mystères de la foi d'une manière rationnelle. Au début de l'ouvrage, il écrit : « quoi qu'il ne soit pas juste d'exiger de la raison naturelle plus qu'elle ne peut, et de l'obliger de nous élever au-dessus de ses forces, si est-ce que par elle-même, il est facile de vous faire voir qu'elle a plus de force et plus de vigueur pour l'établissement des plus hauts mystères de notre religion, qu'elle n'en a pour les combattre, et pour les ébranler ${ }^{7}$.» Sa position nous rappelle Jacques Forton, dit le sieur de Saint Ange. En fait ce dernier, qui manifestait les mêmes arguments à cette époque-là, alléguera de cet ouvrage de Marandé pour justifier son opinion "par autorité » dans la seconde partie de La conduite du jugement naturel publiée en 1641. Il donne aussi les noms comme Jean de Silhon, Yves de Paris, Du PlessisMornay et Raymond Sebond ${ }^{8}$. Le fait que Marandé ait choisi l'anonymat exprime sans doute la peur de se voir reprocher d'avoir fait trop confiance à la force de la raison, c'est ce qui arrivera au sieur de Saint Ange?

Dans les années 1640 l'activité de Marandé comme écrivain devient de plus en plus vive, et on le voit orienter progressivement son œuvre, vers la vulgarisation de la philosophie et la théologie scolastique, notamment celle de Saint Thomas.

En 1641 son nouvel ouvrage apparaît, Le théologien français. Dans cet ouvrage qui est selon l'auteur, dans le prolongement de son Philosophe chrétien, Marandé nous livre des explications en français des notions théologiques, d'un accès difficile. Ce livre, qui a fait l'admiration de Mersenne et de Jean-Pierre Camus, a été dédié en 1641 à l'assemblée générale du clergé attribuant une gratification à l'auteur non sans objections ${ }^{10}$. La préface de ce livre nous éclaire sur le point de vue de l'auteur en même temps pédagogique et apologétique : "Que si le rang qu'ils tiennent dans le monde, ou l'emploi de leurs charges les appelle dans les pays étrangers, et que par les rencontres de la conversation, ils se trouvent engagés à défendre la vérité de leur croyance, contre les sectes différentes qui empoisonnent aujourd'hui la plupart de nos voisins ; leur ignorance ne cause-t-elle pas un scandale à l'église, de la plus haute conséquence? Les ennemis de notre foi en tirent un merveilleux avantage, et se prennent à notre religion, de l'insuffisance et de la faiblesse de celui qui l'entend si peu, et la défend si mal. Après tout il semble que la principale cause de cet abus, procède de l'âpreté des choses qui font partie de cette science que nous appelons théologie, si difficile par la dureté de ses termes, dont les plus subtils se servent pour l'expliquer, qu'elle ne s'est point encore civilisée ni rendue familière et conversable parmi les gens du monde. Ce qui m'a fait résoudre de l'habiller à la française, la dépayser de l'Ecole, et de produire aujourd'hui parmi nous ses excellentes notions, qui jusqu'à présent n'ont paru que sous un habit étranger et peu communicable ${ }^{11}$. $\gg$ Ces paroles du vulgarisateur ne sont pas trop éloignées de l'essai d'explication des mystères de la foi par la raison dans Le philosophe chrétien. Ce dernier était sans doute à l'origine de son inclination vers la vulgarisation théologique.

11 Comme vulgarisation, Marandé publie en 1642 un autre ouvrage intitulé : Abrégé curieux et familier de toute la philosophie. Cet écrit, qui est la présentation en français de la philosophie scolastique, est une sorte de dictionnaire recueillant les commentaires des mots essentiels qui relèvent de chaque partie de la philosophie scolastique, c'est-à-dire la logique, la morale, la physique, la métaphysique et la théologie ${ }^{12}$. Ce livre aurait été reçu avec succès, car, en 1659 , on en trouvera la huitième édition. Il semble que cet ouvrage 
doit beaucoup au livre du sieur de Saint Ange, La conduite du jugement naturel publiée en $1637\left(1^{\mathrm{re}}\right.$ partie) et 1641 ( $2^{\mathrm{e}}$ partie $)$; la question de la relation de ces deux auteurs reste à plus approfondir ${ }^{13}$.

12 En tant qu'ouvrages publiés avant son engagement sur la querelle théologique, nous en connaissons encore trois titres, c'est-à-dire L'esprit de Grenade sur la guide des pécheurs publié en 1645, Morales chrétiennes du théologien français publiées de 1645 à 1648, et La clef ou abrégé de la Somme de Saint Thomas parue en 1649. Ne parlons pas ici du premier, qui est un extrait en français de l'œuvre de Louis de Grenade, dominicain espagnol. Les deux autres sont plus importants pour saisir l'évolution de son œuvre dans sa vie.

Ces deux ouvrages nous offrent l'introduction ou la présentation en français de la théologie de Saint Thomas. Cet essai dont il a eu la conception au plus tard en l'année 1640 , occupait sans doute une place prépondérante dans les œuvres de cette époque ; car l'auteur considérait les autres publications de ces années comme un « divertissement » de son " grand travail » ou ses « études plus sérieuses ${ }^{14}$ ». Au moment de la publication de La clef ou abrégé de la Somme de Saint Thomas en 1649, il manifeste l'intention de poursuivre cette sorte de travail. Mais il sera interrompu dans les années 1650 à 1660 par son intervention dans le débat théologique, qui changera totalement d'orientation. Cependant la polémique n'a pas fait perdre à Marandé son intérêt pour la théologie de Saint Thomas; le travail interrompu dans les années 1640 lui fera écrire dix volumes de plus dans sa dernière œuvre: La clef de Saint Thomas sur toute sa Somme, publiés de 1668 à 1669 , sans doute suite à sa polémique.

N'entrons pas dans le problème du thomisme chez Marandé; bornons-nous à citer quelques paroles de La clef ou abrégé de la Somme, révélant le but de cet ouvrage. En disant qu'il a écrit " pour le secours et le soulagement des apprentis et des novices, qui sortis fraîchement des Ecoles et aguerris dans les exercices de la philosophie, se présentent à l'étude de la théologie ", il parle de la situation actuelle à propos de la théologie de Saint Thomas : « Le désir de savoir est naturel à l'homme, mais les difficultés épineuses dont les plus hautes sciences sont investies, rendent pour l'ordinaire ce désir inutile et sans fruit ; de là vient qu'entre ceux qui s'adonnent à l'étude de la théologie, il s'en trouve si peu qui s'appliquent à la Somme de Saint Thomas : [...] Car que sont tous les commentateurs de la Somme de Saint Thomas, que les truchements de sa doctrine, et les panégyristes de sa gloire? Tellement que la difficulté qu'il y a de se désaltérer en la vive source de la théologie, et la facilité que l'on trouve à s'abreuver dans ses ruisseaux, qui se sont multipliés entre les mains des Docteurs, par le grand nombre de leurs écrits, est à mon avis la raison et la cause, pour laquelle la plupart des écoliers ont peine de s'appliquer à la doctrine de Saint Thomas; et comme le temps destiné au cours de leurs études s'étant écoulé, le soin de leur fortune, ou l'emploi de quelques saintes occupations, qui les appellent ailleurs, ne permet plus qu'ils s'occupent dans un travail si pénible et si laborieux, cela fait qu'ils demeurent frustrés de cette sublime connaissance ${ }^{15}$. » Il va sans dire que le dessein de rendre plus accessible la théologie de Saint Thomas se rattache à celui du Théologien français; dans les années 1640 Marandé se plongeait dans la vulgarisation de la théologie.

Il paraît que par ces travaux de vulgarisateur, Marandé a acquis de la renommée dans une certaine mesure. En 1649 le biographe de Mersenne écrit : « $\mathrm{M}^{\mathrm{r}}$ Marandé, greffier de la cour des aides, assez connu par les livres qu'il a mis en lumière ${ }^{16}{ }^{\prime}$; Adrien Baillet écrit aussi, en parlant de Marandé, greffier : "l'on peut dire que ses livres l'ont fait assez connaître dans le monde ${ }^{17}{ }^{\text {». }}$ 


\section{Relations}

\section{la scène de sa vie se déroule probablement toujours à Paris, où il devait avoir des relations} avec de grands savants de cette époque. 1629, en Angleterre et à Rome comme secrétaire de l'ambassadeur du roi de France ${ }^{18}$. Nous n'avons aucune information sur son séjour à Rome, mais quant à son voyage en Angleterre, nous pourrions l'expliquer par ses relations avec un diplomate nommé Blainville ${ }^{19}$. Ce dernier a été envoyé à Londres par Richelieu comme ambassadeur de l'année 1625 à 1626, mais après son retour en France il a été exilé en Normandie à cause d'un désaccord avec le cardinal. Selon une lettre datée du 26 novembre 1627 envoyée par Blainville à Marandé, celui-ci consolait cet ancien ambassadeur de sa situation en exil, en lui enseignant ses pensées montaigniennes telles que l'on peut lire dans le Jugement des actions humaines ${ }^{20}$.

D'après Richard Popkin, auteur de l'Histoire du scepticisme, Marandé était « le secrétaire de Richelieu $^{21}$ ». Cette thèse est probable, parce qu'outre la dédicace de son premier ouvrage, nous savons qu'il aurait pu être le secrétaire d'un ambassadeur envoyé par Richelieu. Mais il faut reconnaître que nous n'avons pu trouver les sources qui témoignent de leurs relations.

Ses relations avec Descartes sont plus probables: nous ne connaissons pas de choses concrètes au sujet de ces relations, mais Baillet nous a fait découvrir son amitié avec Descartes pendant le séjour de celui-ci à Paris après le retour du voyage en Italie en 1625 jusqu'à son départ en Hollande en $1629^{22}$.

Depuis les années 1630, les relations avec Mersenne ont dû débuter. Hilarion de Coste, biographe de Mersenne, donne la liste des "personnes pieuses et savantes» que ce minime fréquentait, dans laquelle Marandé compte parmi les «illustres écrivains ${ }^{23}$ ", citant de noms Descartes, La Mothe Le Vayer, Gabriel Naudé, etc. Mersenne avait contredit la pensée sceptique déclarée dans le Jugement des actions humaines, mais il a beaucoup apprécié Le Théologien français « qui comprend toutes les difficultés de tous les scolastiques, et qui est faite si élégamment et si dévotement, que chacun l'admire ${ }^{24}$ ». Ajoutons que Mersenne nous dévoile que Marandé était l'un des « excellents joueurs de luth » de cette époque ${ }^{25}$.

21 Il faudrait mentionner aussi les relations qu'il a eu depuis la fin des années 1630 avec Jean-Pierre Camus, l'évêque de Belley. De temps en temps ils se sont vus et ont discuté sur la question de la grâce ${ }^{26}$. Nous savons que Marandé lui a adressé ses ouvrages tels que Le philosophe chrétien et Le théologien français, et a reçu des lettres pleines d'éloges ${ }^{27}$. Il est intéressant de savoir que l'évêque de Belley, qui arbitrera l'affaire de Saint Ange en faveur de Jacques Forton, avait déjà lu et apprécié Le philosophe chrétien qui présentait une opinion semblable à celle de l'auteur de La conduite du jugement naturel.

\section{Professions}

Passons à sa position sociale. Comme nous l'avons mentionné, Marandé a commencé à travailler comme greffier de la cour des aides de Paris au plus tard en l'année 1641, mais 
sans doute était-il dans cette position jusqu'à 1649. Au début de l'année suivante, il est déjà aumônier du roi, et il semble qu'il a touché des gages s'élevant à 300 livres $^{28}$. En 1651 on le verra s'intituler "conseiller et aumônier du roi $^{29}$ ". Quelque temps après ce changement de position sociale, il changera aussi la direction de son œuvre pour s'engager dans un débat théologique. Au début des années 1650 nous constatons donc d'un côté le passage de l'homme de justice à l'homme de cour, et de l'autre côté le passage du vulgarisateur au polémiste.

Après tout, dans les années 1640 tout en travaillant dans la cour des aides, Marandé rédigeait ses ouvrages à ses heures de loisir : il écrit en 1652 lors de la publication de la troisième édition du Théologien français: «La troisième édition de cet ouvrage m’a rencontré dans un temps où j'avais un peu plus de loisir que dans les deux autres précédentes; ce qui m'a donné sujet de le revoir, de le renouveler et de l'accroître en beaucoup de lieux, [...]. Mes occupations ordinaires m'ont laissé si peu de temps par le passé que je puis dire que les grands ouvrages que j'ai donnés au public depuis dix ou douze ans, se sont échappés de ma main après le premier trait de pinceau. Le succès favorable qu'ils ont trouvé m'oblige de les revoir pour leur donner par une seconde touche, plus de perfection et de politesse qu'ils n'en ont eu dans leur première naissance ${ }^{30}$ ». Il parait que le changement de position sociale lui a octroyé plus de temps et de liberté pour se pencher sur ses travaux d'écrivain.

En fait le titre d'aumônier du roi, qu'il a probablement acheté par le truchement d'offices rémunérés, était un titre honorifique n'impliquant pas de fonctions : les aumôniers qui servaient pratiquement la Maison du roi sont appelés "aumôniers servants » ou " aumôniers ordinaires », mais il est vrai qu'il y avait, en plus, de nombreux aumôniers n'ayant pas leurs fonctions. Par exemple, Guillaume de Peyrat, auteur de L'histoire ecclésiastique de la cour, écrit en 1645 à la fin d'un chapitre consacré à la description des aumôniers: "Je finirai ce chapitre par les Aumôniers honoraires; car sur l'état de la Chapelle du Roi, il y a un grand nombre d'Aumôniers, pour les honneurs et privilèges seulement, où sont employés plusieurs Archevêques, Évêques, Abbés et autres bénéficiers qualifiés ainsi par un état du paiement que le Roi a ordonné être fait à ses officiers [...] à commencer du $1^{\text {er }}$ Juillet 1570 et à continuer jusqu'à ce que sa Majesté ait fait nouvel état, lequel se trouve au Greffe de la Cour des Aides. Il y avait dix-sept Aumôniers ordinaires qui servaient par quartier, et cent trente-deux Aumôniers, qui étaient sans doute des Aumôniers honoraires ${ }^{31} »$. Dans la liste des officiers de 1650, nous découvrons 9 aumôniers ordinaires et 132 aumôniers honoraires dont Léonard de Marandé.

Nous ne savons pas exactement dans quelle situation il a obtenu sa nouvelle position, mais cet avancement n'était certainement pas la carrière qu'on faisait alors ordinairement ; nous le savons grâce à la description de Baillet (1649-1706), car il a écrit curieusement: "Pour M. de Marandé l'on peut dire que ses livres l'ont fait assez connaître dans le monde. Mais il faut prendre garde de ne le pas confondre avec un ecclésiastique de même surnom et du même temps. Celui-ci se nommait Léonard de Marandé, se qualifiait Conseiller et Aumônier du Roi, et se mêlait de théologie. Mais l'ami de Descartes était greffier de la cour des aides, et donnait le reste que lui laissait son office à des traductions françaises, et à des exercices de philosophie et de mathématique ${ }^{32}$ ». Le témoignage de Baillet nous offre de précieuses informations sur Marandé, mais il nous faut écarter son point de vue qui suppose l'existence de deux personnes nommées Léonard de Marandé, car ce biographe a séparé totalement, par erreur, les deux parties de la vie de Marandé. Nous n'avons pas le temps de vérifier cette erreur, mais il est vrai 
qu'elle est confirmée par d'autres témoignages plus fiables comme ceux d'Arnauld et Hermant $^{33}$. Le biographe de Descartes, né en 1649 et devenu l'un des ami de Port-Royal sous l'influence de Godefroi Hermant ${ }^{34}$, devait entendre dire le nom de Marandé antijanséniste, conseiller et aumônier du roi, mais il n'a pu imaginer que ce polémiste était la même personne que l'officier de la cour des aides, qui consacrait ses loisirs à la traduction, philosophie et mathématiques, tant ces deux carrières étaient difficiles à s'unir l'une à l'autre dans la vie d'une même personne.

\section{Marandé ennemi de Port-Royal}

26 Maintenant passons à l'époque de sa polémique pour examiner la deuxième partie de sa vie. Après la promotion sociale dont nous avons parlé précédemment, nous le voyons changer aussi radicalement de direction dans son écrit, c'est-à-dire qu'il intervient dans l'affaire janséniste. Nous allons d'abord examiner la situation de ce changement, et puis nous étudierons ses écrits polémiques ainsi que ses relations pendant le débat.

\section{Du vulgarisateur au polémiste}

La polémique de Marandé naît de son écrit comme vulgarisateur. Un ouvrage de Marandé publié en 1652 intitulé Antiquités de l'Église de l'ancien usage des sacrements nous donne la clef pour comprendre cette situation. Ce livre est le premier qu'il a publié comme conseiller et aumônier du roi. Mais il me semble que cet ouvrage n'est pas rédigé en tant qu'écrit polémique. A la différence de ses écrits anti-jansénistes qui attaquent avec persistance Arnauld et Port-Royal, leurs noms ne se trouvent même pas dans cet ouvrage consacrant tous les discours à la description historique des cérémonies autour de trois sacrements : le baptême, la confirmation et la pénitence.

Il est vrai que la préface de cet écrit nous permet de comprendre que tout en ayant conscience du différend soulevé par la Fréquente communion (1643), l'auteur situe son ouvrage dans le prolongement de ses travaux des années 1640 comme vulgarisateur, et prend soin de ne pas entrer dans le débat entre les «savants » : il écrit : "[...] Ayant considéré que le différend qui s'était mu entre les doctes, ne regardait proprement qu'une pratique particulière revêtue de quelques circonstances, et non pas la matière générale, qui regarde le gros des Sacrements, leurs cérémonies et leurs anciens usages, tels qu'ils ont été autrefois pratiqués dans l'Église; J'ai cru que je pouvais continuer ma pointe et qu'un si riche trésor, ne devait point être caché dans un temps, où il me semble que les esprits lassés de la lecture des Romains, et des folles vanités d'une antiquité païenne, ne recherchent plus que les vieilles médailles d'une sainte antiquité, ensevelies dans les tombeaux des SS. Pères, je veux dire dans leurs écrits. [...] Et d'ailleurs, toutes personnes indifféremment ne sont pas capables de comprendre et de concevoir les hautes vérités du Christianisme, qui consistent en une spéculation très éminente ; mais tous peuvent goûter et savourer les choses pratiques, en leur développant les mystères qui se trouvent enclos sous l'écorce des cérémonies anciennes et extérieures, dont l'Église a revêtu nos Sacrements, pour rendre en quelque façon, sensible et maniable, ce qui est de soi invisible et incompréhensible à l'esprit humain. C'est ce que nous essayerons d'effectuer [...]. / Mais parce que le changement des usages et des cérémonies dans l'administration des saints mystères, a fait cabrer quelques hérétiques, et qu'il pourrait causer quelque scrupule dans les faibles, qui pour n'être pas capables de discerner le corps, d'avec son 
vêtement, ni la substance d'une chose, d'avec ses accidents, se persuadent que ce ne sont plus les mêmes sacrements, $[. . .]^{35}$.» Marandé n'est pas encore polémiste. Ces paroles sont vraiment celles du vulgarisateur et nous rappellent ce qu'il voulait faire dans Le théologien français.

Cependant la position fondamentale adoptée dans cet ouvrage est évidemment celle des jésuites, et en ce qui concerne la pénitence qui en est le sujet central, on trouve des arguments qui se heurtent clairement à la Fréquente Communion. Pour comprendre ce point-là, il suffit d'énumérer les titres de quelques discours: Traité 7, Discours $4:$ « De ceux qui dans la persécution reniaient la Foi, et le nom de Jésus-Christ, et que parfois ils communiaient avant que d'avoir achevé leur pénitence»; Traité 13, Discours 1: "Que selon l'ordre de Dieu qui nous est marqué dans l'ancienne loi, la rémission des péchés a toujours précédé la satisfaction du pénitent »; Traité 14, Discours 2 : «Que la satisfaction n'est pas la plus importante des parties de la pénitence ${ }^{36} »$. Il est vrai que ce livre de Marandé contient des points qui seront remis en question dans la critique de la morale jésuite dans les Provinciales $^{37}$.

30 À la fin du compte, cet ouvrage, tout en étant rédigé du point de vue du vulgarisateur, a pour effet de déclarer, bien que tardivement, son adhésion à la cause des jésuites sur la question évoquée dans le débat autour du livre d'Arnauld. Par les Antiquités de l'Église, Marandé n'a pu s'empêcher de s'attirer l'inimité de Port-Royal, car Hermant écrit : «Le sieur de Marandé, autrefois commis au greffe de la cour des aides, s'échauffant tous les jours de plus en plus pour la cause de jésuites, composa au mois de février de cette année 1652, un livre intitulé : Les antiquités de l'Église en l'usage des sacrements, principalement celui de la pénitence: contre les erreurs du livre de la fréquente communion ${ }^{38}$. " Dans cette citation, c'est Hermant qui a ajouté par erreur au titre de l'ouvrage de Marandé : «contre les erreurs du livre de la Fréquente communion». Cet ouvrage se révèle être un facteur entraînant Marandé du vulgarisateur au polémiste ; en effet, juste après la publication de ce livre, une réponse est apparue du côté de Port-Royal sous le titre: Les saints pères de l'Église vengés par eux-mêmes des impostures du sieur de Marandé dans son livre des Antiquités de l'Église (1652), et c'est par les réfutations de cette réponse que Marandé prend la plume contre Port-Royal pour la première fois. À partir de ce moment-là, il changera de direction et n'hésitera plus à entrer dans le débat théologique « entre les doctes ».

31 Ajoutons que le polémiste ne s'éloigne pas du vulgarisateur; car bien que le point de vue de celui-ci soit plus pédagogique que polémique, nous avons constaté que le but de l'auteur du Théologien français était aussi apologétique, et en plus, dans les Antiquités de l'Église, Marandé avait en tête les hérésies comme Calvin et Luther.

\section{Ecrits polémiques des années 1650 à 1660}

La plupart des ouvrages postérieurs aux Antiquités de l'Église sont consacrés aux critiques sévères contre Port-Royal, hormis deux de ses ouvrages : Le berger fidèle publié en 1661, traduction française d'un poème italien de Jean-Baptiste Guarini (1538-1612) : Pastor fido, et son dernier ouvrage La clef de Saint Thomas sur toute sa Somme publié de 1668 à 1669.

33 Nous confirmons la nature de ses écrits polémiques. En 1653 Marandé publie en même temps deux ouvrages contre Arnauld: l'un est intitulé: Pénitence publique d'un illustre janséniste adressé à Monsieur Arnauld, l'autre : Inconvénients du Jansénisme adressé à Monsieur Arnauld. Dans le premier qui est la réfutation de la réponse aux Antiquités de l'Église, l'objet de la critique est toujours Arnauld considéré comme auteur de la réponse ; alors que dans 
le second, bien que le destinataire soit également Arnauld, la cible est l'ensemble d'une secte appelée « jansénisme », dont l'auteur souligne le danger pour l'église.

L'année suivante a paru un autre ouvrage sous un titre semblable: Inconvénients d'État procédant du jansénisme. Dans ce livre, l'auteur entreprend de démontrer que le « jansénisme » est dangereux pour l'ordre de l'État. Par exemple, il écrit : « La religion et l'État dans un empire chrétien, sont unis et liés d'une étreinte si ferme, qu'il est impossible que quelque nouveauté donne atteinte à la Religion, et divise le cœur de ses sujets, par des sentiments différents; qu'elle ne blesse en même temps le corps de l'État, et n'en partage l'unité ; parce que les sujets de l'Église, sont les sujets de l'État. C'est la raison pour laquelle toute secte en matière de Religion, est toujours une secte d'État. Mais ce que la secte du Jansénisme a de particulier, et qui est en elle comme un caractère spécial, qui la distingue de toutes les autres, est qu'elle est plus une secte d'État, que de Religion $^{39}$. »Comme il situe l'origine de ce danger au niveau politique dans le pamphlet de Jansénius publié en 1635, Mars Gallicus, l'auteur consacre un des articles à le réfuter ${ }^{40}$.

Il ne faut pas oublier que Marandé a dédié cet ouvrage au roi Louis XIV. En soulignant le danger politique causé par le "jansénisme », l'auteur essaie d'inciter le pouvoir politique à exécuter la condamnation décidée par le pape, car il écrit dans l'épître dédicatoire au roi : «Sire, le saint Père à l'exemple des autres papes qui l'avaient devancé dans la chaire de saint Pierre, a depuis peu à la prière de Votre Majesté, et à la poursuite des évêques de France, interposé son décret, et condamné d'hérésie une doctrine profane tant de fois fulminée par les papes et par les conciles, et dont la nouveauté menaçait l'Église de désolation et de schisme, et votre État de troubles et de division. Il ne reste donc plus à la gloire de terminer par le bras de sa puissance, ce qu'elle a si heureusement commencé pour donner la paix à l'Église, et le repos à ses peuples; faisant à l'imitation de ses prédécesseurs (dont la mémoire sera toujours glorieuse), exécuter dans la France les Décrets du S. Siège ${ }^{41}$.» Il me semble que l'originalité du point de vue de cet ouvrage a permis à l'auteur de laisser plus ou moins son nom dans l'histoire de la querelle janséniste: aujourd'hui quand on traite par exemple du problème «Jansénisme et politique », on cite souvent ce livre de Marandé.

L'une des pièces ajoutées à la fin des Inconvénients d'État ne contribue pas moins à l'attention prêtée à cet ouvrage : il s'agit de la Lettre circulaire à Messieurs les disciples de Saint Augustin. Cet écrit diffusé en manuscrit par un des ennemis de Port-Royal sous forme d'une lettre circulant parmi les amis de Port-Royal, a pour but de dévoiler plusieurs moyens que ceux-ci employaient pour accroître leur force. C'est sans doute une sorte de calomnie, mais il a eu tellement d'influence, qu'Arnauld se plaint à plusieurs reprises de l'injustice de cette lettre et que Pascal en fait mention dans la $15^{\mathrm{e}}$ Provinciale ${ }^{42}$. Marandé a été le premier à publier cette lettre manuscrite, imprimée plusieurs fois jusqu'à la fin du XVIII ${ }^{e}$ siècle $^{43}$.

En 1655 Marandé s'applique plus qu'avant à combattre Port-Royal. Nous savons qu'il a publié cette année-là quatre ouvrages.

38 Aussitôt après la sortie en février de la Lettre à une personne de condition (première lettre) d'Arnauld, il a publié la Réponse à la lettre de Monsieur Arnauld. Ce pamphlet était un extrait de la préface d'un autre ouvrage qu'il préparait en même temps et qu'il publiera l'année suivante, intitulé : Règles de Saint Augustin pour l'intelligence de sa doctrine.

En juillet il a abordé la traduction du bref d'Innocent $\mathrm{X}$ envoyé à l'assemblée des prélats le 29 septembre 1654. Ayant précisé que le pape avait condamné dans sa bulle Cum occasione 
la doctrine de Jansénius, ce bref avait un sens définitif pour la question de fait, et la traduction française de ce bref latin avait paru en juin $1655^{44}$, mais selon l'avis mis en exergue de la traduction de Marandé, c'était une traduction favorable à Port-Royal. C'est pour mettre en valeur et révéler les défauts de cette traduction et pour les corriger qu'il a publié, un mois après, la nouvelle traduction, intitulée : Traduction fidèle du dernier décret d'Innocent X portant condamnation de la doctrine de Jansénius et des livres faits pour sa défense (chez G. et $\mathrm{S}$. Cramoisy, avec le privilège du roi) ${ }^{45}$. Dans cette publication, Marandé présente en trois colonnes le texte original du décret en latin, l'ancienne traduction appelée " défectueuse », et sa nouvelle traduction « fidèle », pour qu'on puisse en faire la comparaison. La traduction de Marandé sera adoptée dans le procès-verbal de l'assemblée générale du clergé, publié l'année suivante ${ }^{46}$.

La sortie de la Seconde lettre d'Arnauld au début de septembre de cette année a amené Marandé à répondre immédiatement, et il a publié au mois suivant la Réponse à la seconde lettre de Monsieur Arnauld. Aussitôt que l'examen de la Seconde lettre a été décidé en Sorbonne en novembre, Marandé prend sa plume même pour répondre à un écrit présenté à l'assemblée de Sorbonne par Arnauld pour sa défense : le pamphlet qu'il a ainsi publié en décembre est intitulé : Réponse à l'écrit que Monsieur Arnauld a fait présenter aux docteurs de la Sacrée Faculté de théologie, assemblés en Sorbonne pour la censure de sa Seconde lettre.

41 En 1656, l'attaque de Marandé contre Arnauld et Port-Royal se poursuit avec autant de vigueur et de promptitude que les années précédentes. Tout de suite après la décision de la censure définitive de l'ouvrage d'Arnauld à la fin de janvier, il s'est attelé à un nouvel ouvrage et l'a publié en février sous le titre: Réflexion sur la censure que les docteurs de la sacrée faculté de théologie assemblés en Sorbonne, ont faite de la seconde lettre de Monsieur Arnauld. L'auteur se félicite de la condamnation d'Arnauld, et déclare que sa réponse a été loyale, suite à la censure de la lettre. En parlant de la censure, il écrit : « Elle est aussi la perfection de ma Réponse, parce que se trouvant fortifiée du secours de cette Censure, elle fait voir plus clairement à tous les sages, et à toutes les personnes désintéressées, qu'elle a été la sincérité de mes intentions, dans le dessein que j'ai eu de défendre l'intérêt de la religion et de l'État, contre les erreurs et les fausses maximes de cette seconde Lettre 47. »

Cet écrit est un petit pamphlet qui ne compte que quatorze pages, tandis qu'un autre ouvrage publié à la même époque est un livre volumineux qui comporte plus de cinq cents pages; Il est intitulé : Règles de Saint Augustin pour l'intelligence de sa doctrine, avec la réfutation des principes de Jansénius par eux-mêmes et par Saint Augustin. Comme nous l'avons dit, une partie de cet ouvrage a été déjà publiée en réponse à la première lettre d'Arnauld.

$\mathrm{Au}$ mois de mars, Marandé publie encore un autre ouvrage dont le titre est un peu long: Considérations sur un libelle de Port-Royal intitulé Défense de la constitution d'Innocent X. etc., sur la retraite des jansénistes, sur la protestation de Monsieur Arnauld et sur les Lettres qu'il a fait courir dans Paris, depuis la censure de Sorbonne $e^{48}$. L'une des cibles de la critique de l'auteur est les quatre premières Provinciales qui venaient de paraitre. La réponse de Marandé est toujours rapide, parce que c'est la deuxième tentative de réponse aux Provinciales, qui suit une lettre anonyme parue le mois précédent ${ }^{49}$. Marandé considère Arnauld comme l'auteur des Petites Lettres, en parlant d'un écrit d'Arnauld présenté à la Sorbonne en guide de protestation, il écrit : « pour lui donner plus de maturité, il l'a nourri et fortifié par la chaleur de quelques écrits satyriques, entre autres de quatre Lettres adressées à un Provincial, qui des matières les plus importantes de la foi, en font des matières de raillerie 
dans la bouche des peuples; ayant imité en cela le procédé des hérétiques anciens et modernes, qui pour énerver les censures qui les ont foudroyé, ont fait un jeu et une raillerie de leur propre condamnation »; «Que si l'auteur de ces Lettres se trouve joué et raillé dans les réponses que j'y faits, qu'il se l'impute à soi-même, puisqu'il nous a ouvert cette carrière, et que celui qui se joue des choses divines, pour les exposer au mépris, mérite bien qu'on se raille de sa personne, et qu'on n'en fasse pas grand état ${ }^{50}$. » Nous n'avons pas le temps de présenter les remarques qu'il a faites pour chaque lettre, mais il est vrai que l'un des arguments présentés à l'appui de sa critique est le problème de la raillerie remis en question dans la $11^{\mathrm{e}}$ Provinciale; dans un autre endroit, il dit aussi : "Quant au premier point qui concerne la bouffonnerie de cet auteur, j'avoue que s'il s'était mêlé de monter sur le théâtre, et d'en faire profession, il y aurait peut-être mieux réussi, qu'à débiter des doctrines nouvelles; ou en tout cas s'il avait employé ses railleries sur une matière indifférente, il serait assez divertissant par ses plaisanteries. Mais de voir qu'un chrétien, qu'un prêtre, et un docteur, qui s'était vanté autrefois d'avoir juré sur l'autel des martyrs de défendre les vérités de l'église, et de les respecter inviolablement jusques à l'effusion de son propre sang: de voir, dis-je, que cet homme qui passe maintenant dans l'esprit de l'Église pour un opiniâtre, pour un endurci dans l'erreur, et pour un hérétique retranché de la communion de la Faculté de théologie de Paris, et dépouillé de la qualité de docteur, soit le premier à rire de soi-même et de son propre malheur, à railler de sa disgrâce, et à divertir les autres de son désastre par ses bouffonneries, dans un temps où son cœur, ses yeux et sa bouche, devraient être remplis de sanglots, de larmes et de soupirs, c'est une chose bien étonnante, et une marque assurée en sa personne, ou d'un entier oubli de son salut, ou, qui pis est, d'une impiété parvenue au plus creux de l'abîme, où l'on méprise toutes choses. Mais qu'il se ressouvienne qu'on ne se raille point des choses divines, qu'on ne se moque point de la doctrine de l'Église; en un mot qu'on ne se joue pas de Dieu (comme parle la sainte Écriture) sans attirer sur soi le dernier anathème de la justice divine ${ }^{51}$.» Comme dans cet écrit, Marandé considère injustement la plupart des écrits sortis du côté de Port-Royal comme provenant de la main d'Arnauld, mais il me semble que cela vient aussi de sa stratégie de la polémique, ce que nous verrons plus tard.

Marandé semblait ainsi s'adonner au mouvement anti-janséniste, mais curieusement après ces ouvrages de 1656 il ne publie aucun écrit jusqu'à l'année 1661. Il est vrai que durant cette période, nous n'avons aucun document qui fasse mention de Marandé. Nous savons seulement qu'il recommencera avec autant d'ardeur qu'avant, à publier les écrits contre Port-Royal en 1661 en même temps que le problème janséniste, momentanément apaisé, apparaît de nouveau sur le devant de la scène.

Un nouvel ouvrage de Marandé concerne la signature du formulaire. Toute de suite après la prescription de la signature par l'assemblée du clergé en février 1661, il a dû se mettre à un écrit intitulé : Question de fait touchant Jansénius, traité par le droit et par le fait : avec la réponse à tous les libelles de Port-Royal, qui ont paru depuis deux ans. Le privilège du roi lui a été octroyé pour cet ouvrage, une semaine après la décision du Conseil d'État, faite le 13 avril pour ordonner la signature. Cet ouvrage contient aussi les réponses aux écrits de Lalane, Arnauld et Nicole ${ }^{52}$.

Juste après l'impression de cet ouvrage, Marandé a achevé un autre petit écrit intitulé : Réflexions sur une lettre manuscrite qui parait sous le nom de Monseigneur l'Évêque d'Angers. C'est la réfutation d'une lettre d'Henri Arnauld adressée au roi datée du 6 juillet 1661, car selon l'auteur, «cette lettre était une lettre supposée, sous le nom de Monseigneur 
l'Évêque d'Angers. [...] on pouvait plus justement attribuer cette lettre aux Docteurs de Port-Royal, qui cent et cent fois ont dit les mêmes choses qui se lisent dans cette Lettre, que non pas au Prélat, sous le nom duquel elle se laisse voir parmi nous ${ }^{53}$.» Ainsi que dans La question de fait, le point du débat consiste à savoir si la question de fait peut constituer une hérésie. Antoine Arnauld publiera en même année deux écrits pour défendre son frère ${ }^{54}$; il n'a fait aucune mention du nom de Marandé, mais celui-ci y a répondu tout de suite par un ouvrage : Réponse à deux libelles de MM. de Port-Royal contre les réflexions sur une lettre d'Angers au roi.

En même temps que la critique de la lettre de l'évêque d'Angers, Marandé préparait un autre ouvrage plus volumineux, publié en 1663 sous le titre: Défense de l'Église contre un écrit de Port-Royal, intitulé Lettre sur la constance. C'est la réponse à l'écrit, d'un ami de PortRoyal, Guillaume Le Roi, abbé de Hautefontaine (1610-1684) ${ }^{55}$.

Grâces aux témoignages de Jean Ferrier et de Marandé lui-même, nous savons également qu'en 1662 il a pratiquement achevé un ouvrage dont le titre est L'infaillibilité du pape ${ }^{56}$, et qu'en 1664, pour la défense du mandement de Hardouin de Péréfixe l'archevêque de Paris, il préparait deux autres écrits anti-jansénistes dont l'un est intitulé : la Foi problématique des docteurs de Port-Royal ${ }^{57}$. Cependant nous savons peu de choses sur ces écrits que nous n'avons pas encore trouvés.

Il est difficile de suivre Marandé après l'année 1664. Sans doute a-t-il persisté dans son mouvement anti-janséniste, mais à un certain moment il a dû revenir à ses travaux comme vulgarisateur, travaux interrompus depuis la fin des années 1640 ; ainsi, de 1668 à 1669 il publie sa dernière œuvre : La clef de saint Thomas sur toute sa Somme. En 1676 est sortie la quatrième édition du Berger fidèle, traduction dont on a fait mention, avec les augmentations et corrections apportées par Marandé. C'est la dernière trace, et nous n'avons donc aucune information sur sa mort.

\section{Marandé et les jésuites}

50 Il faudrait ensuite enquêter sur ses relations pendant la polémique, car Marandé n'a pas développé sa campagne anti-janséniste tout seul. En fait quelques-uns de ses écrits sont de toute évidence ceux qui étaient commandés, et il est arrivé qu'il fût pressé de publier son écrit le plus tôt possible. Ce qui sous-entend une coopération sous-jacente avec d'autres anti-jansénistes. Mais il faut d'abord reconnaître qu'il n'est pas facile de saisir les rapports entre les ennemis de Port-Royal qui existaient en Sorbonne, dans l'assemblée du clergé ou des prélats, dans les ordres religieux, dans le pouvoir politique, dans les milieux juridiques etc. Il en va de même pour le cas de Marandé; néanmoins ses rapports avec les autres ennemis de Port-Royal sont à examiner d'autant plus qu'on ne voit pas clairement l'appartenance sociale de sa position « conseiller et aumônier du roi ».

51 Il est au moins certain que l'un de ses principaux collaborateurs est la Compagnie de Jésus. Il a lu les écrits des jésuites publiés contre Port-Royal et a appris les points de leur critique : il nous renvoie souvent aux écrits de François Annat et François Pinthereau; il arrive qu'il adopte l'argument de Jean de Brisacier ${ }^{58}$.

Le lien avec les jésuites se révèle dans une lettre adressée à Marandé par Jean Ferrier daté du 5 juillet 1662. Ce jésuite, polémiste anti-janséniste étant lors à Toulouse, sera en 1670 confesseur du roi, prenant la succession du Père Annat. Il écrit : «J'ai parlé à un libraire de cette ville pour savoir s'il voudrait entreprendre d'imprimer votre ouvrage de l' 
Infaillibilité du pape. Il a accepté avec plaisir, et je lui ai promis de lui avoir la permission et les approbations nécessaires. Une chose lui pourrait faire de la peine, c'est le défaut de privilège de $\mathrm{M}$. le chancelier. Car il y a sujet de craindre que, n'ayant pas de privilège, on réimprimera votre ouvrage à Paris ou à Lyon, et le libraire qui l'aura imprimé se trouvera frustré par ce moyen du fruit de son travail. Possible qu'il se pourra trouver quelque remède à cet inconvénient. Je ne doute pas que votre ouvrage ne soit de grand débit. Votre réputation est si grande dans toute la France et dans les pays étrangers, que toutes les personnes intelligentes seront ravies d'avoir une pièce sur un sujet de cette importance qui soit de votre main. C'est le dernier refuge des jansénistes que de révoquer en doute l'autorité du Saint Siège, et si on les chasse de ce fort, je ne sais s'ils trouveront quelque autre chicanerie pour autoriser leurs erreurs ${ }^{59}$.» Ferrier, qui semble se charger d'imprimer un ouvrage de Marandé sur l'infaillibilité du pape, cherche non seulement un imprimeur mais aussi la permission et les approbations. Ce qui nous autorise à présumer que les jésuites assuraient la publication de ses ouvrages qui auraient été utiles pour leur politique.

Après avoir informé Marandé sur le litige d'alors, c'est-à-dire sur l'infaillibilité du pape, ce jésuite finit sa lettre en écrivant : «Continuez donc, Monsieur, à travailler contre eux pour la défense d'une des plus importantes vérités du christianisme; et si vos travaux ne travaillent pas à leur conversion, ils serviront pour affermir les fidèles dans la créance des vérités catholiques, et leur fourniront des armes pour se défendre contre les surprises des hérétiques. Je vous supplie de compter sur moi, et que je suis [...] votre intime ami ${ }^{60}$.» Ferrier estimait tellement la force de la plume de Marandé comme polémiste, qu'il l'a présenté à Louis XIV en $1663^{61}$. De toute façon on peut dire que les jésuites voulaient profiter, dans leur polémique, de la virulence de son style. Ce n'est pas sans raison qu'Hermant a écrit que Marandé était l'« écrivain à gages des jésuites». Il arrive qu'ils fassent paraître les écrits de Marandé à la fin de leurs livres : c'est le cas de l'ouvrage du Père Annat: Réponse à quelques demandes dont l'éclaircissement est nécessaire au temps présent $(1656)^{62}$, qui reproduit deux pamphlets de Marandé parus juste avant cet écrit ${ }^{63}$.

En ce qui concerne l'utilisation de ses œuvres, ajoutons quelques autres cas. En 1666 un ouvrage est sorti sans doute aux Pays-Bas sous le titre : L'origine est les causes du jansénisme avec certains règlements et instructions. C'est un extrait du livre des Inconvénients d'État publié en 1654. Selon Arnauld, il a été publié par ses adversaires pour servir le mouvement anti-janséniste ${ }^{64}$. Il arrive que cet extrait ait été ajouté à la fin du livre de Pinthereau: La naissance du Jansénisme (1654). En 1675, en même temps qu'a paru une traduction néerlandaise de cet extrait ${ }^{65}$, nous verrons apparaitre un autre extrait des Inconvénients d'État : il est intitulé : Bouclier contre les nouvelles erreurs, tiré de la doctrine de St. François de Sales... et proposé par le sieur Marandé (s.l.) ${ }^{66}$. Le livre des Inconvénients d'État survivra dans le contexte anti-janséniste au XVIII ${ }^{e}$ siècle : en 1791 nous voyons le résumé de ce livre présenté dans un article de la revue : Journal historique et littéraire ${ }^{67}$; le sujet de cet article est « l'histoire de la naissance de la plus détestable secte qui ait encore ravagé l'Église de Dieu ${ }^{68}$. » Le livre de La question de fait publié en 1661 sera aussi utilisé en 1680 à Cologne pour composer un livre intitulé : Quaestio facti quam appellare hodie placuit, livre en français malgré le titre latin, contenant les écrits du même sujet du Père Annat et de Denis Amelote (1606[9 ?]-1678), oratorien et adversaire de Port-Royal ${ }^{69}$. Nous ne savons pas exactement qui a utilisé ainsi les écrits de Marandé, mais il est certain que les ennemis de Port-Royal ont essayé de tirer parti de ses écrits polémiques. 


\section{Polémique et gloire}

Suite à ces attaques répétées, Marandé mérite bien l'appellation "polémiste antijanséniste farouche». Cependant pour les gens de Port-Royal il était plutôt l'objet d'aversion que l'adversaire à combattre; Hermant l'appelle, par exemple, «l'un des plus faibles et des plus ridicules écrivains de notre siècle" ou "l'homme méprisé et méprisable ${ }^{70} »$. Certes il n'est pas étonnant que la polémique engendre l'aversion, puisqu'il est vrai que parmi les écrits lancés contre Port-Royal se trouve de pures calomnies. Mais leur aversion pour Marandé est tellement forte qu'il me semble exister, à l'origine de ce sentiment, d'autres éléments que le débat théologique. Ils nous conduiront de nouveau dans la vie de Marandé. Pour examiner ce point-là, nous allons essayer, en dernier lieu, d'examiner les attitudes réciproques de Port-Royal et de Marandé dans leur polémique.

\section{Théologien amateur}

56 Ces attitudes se manifestent dès le début de la polémique, c'est-à-dire dans la situation autour de la publication des livres qui suivent les Antiquités de l'Église.

57 Comme nous l'avons vu, ce livre de Marandé publié en 1652 contenait des arguments se heurtant complètement au livre d'Arnauld. Ce dernier n'y a pas répondu, mais dans la même année une réponse intitulée : Les saints Pères de l'Église a paru sous le nom de Sainte Anne, attribué à quelques amis de Port-Royal ${ }^{71}$; mais cette réponse ne s'adresse pas à Marandé mais à Claude Morel, docteur en Sorbonne et adversaire passionné de Port-Royal ${ }^{72}$. Celui-ci n'y a pas répondu, et l'année suivante Marandé a publié deux ouvrages pour y répondre, c'est-à-dire La pénitence publique d'un illustre janséniste et les Inconvénients du Jansénisme, qui sont, à leur tour, adressés à Antoine Arnauld. Dans cet échange, nous pouvons constater un certain décalage entre l'objet de la critique et le destinataire : pour répondre au livre de Marandé, Sainte Anne a écrit à Claude Morel ; et pour répondre au livre de Sainte Anne, Marandé a écrit à Arnauld, qui n'en était pas cependant l'auteur. Il me semble que ce décalage nous permet de saisir la modalité fondamentale de la polémique entre Marandé et Port-Royal.

58 En expliquant la raison pour laquelle il a écrit à Claude Morel, non pas à Marandé, l'auteur des Saints Pères de l'Église dit: «J'avais pensé d'abord de m'adresser au même Auteur, afin de lui montrer ses erreurs: Mais comme j'ai appris, que sa profession n'était pas celle de théologie, et que n'ayant été nourri jusqu'à maintenant dans le Palais, et n'ayant appris qu'un peu de Scolastique depuis quelques années qu'il a mise en français, et où il $\mathrm{y}$ a beaucoup à redire, et un grand nombre de contradictions qui peuvent être connues des moins clairvoyants; j'ai cru certainement qu'il n'avait jamais lu les livres qu'il citait : qu'il n'avait travaillé que sur de faux mémoires, ou sur la foi de quelques livres censurés ; Car autrement je le tiens trop homme d'honneur, pour avoir osé se servir contre ces vénérables maîtres de l'antiquité, de tant d'impostures et de mensonges dont il ne faut que des yeux pour le convaincre. Ainsi je n'aurais rien profité de m'adresser à lui pour l'éclaircissement des choses qu'il ne sait $\operatorname{pas}^{73}$. » Si l'auteur n'a pas écrit à Marandé, c'est parce que celui-ci était ignorant en théologie, et cette ignorance est attribuée à sa carrière, c'est-à-dire au fait qu'il était greffier de la cour des aides de Paris. Sans doute est exprimé ici un certain mépris pour Marandé, théologien amateur, dont la «profession 
n'était pas celle de théologie». Quant aux ouvrages théologique qu'il a publié en travaillant dans la cour des aides, loin d'étaler sa formation théologique, ils ne servent qu'à prouver qu'il est amateur qui, en étant officier profane, s'est mêlé de théologie qui n'était pas son domaine. De plus, ses ouvrages dans les années 1640 étaient d'autant plus méprisables pour Sainte Anne que c'étaient des travaux de vulgarisateur, car la vulgarisation est voisine de l'amateurisme. Après tout, ce mépris exprimé par l'auteur des Saints Pères de l'Église est commun aux réactions des amis de Port-Royal : ils suivront donc l'attitude de cet auteur qui a évité de discuter avec Marandé.

D'autre part, pour réfuter le livre des Saints Pères de l'Église dont l'auteur n'était pas exactement connu, Marandé adresse ses réponses à Antoine Arnauld. Il commence son « avis à $\mathrm{M}^{\mathrm{r}}$ Arnauld » ajouté en exergue de La pénitence publique par les paroles suivantes : "Monsieur, j'ai reçu de votre part le livre injurieux que vous et les vôtre avez fait contre moi, et contre les livres des Antiquités de l'Église, que j'ai donné au public depuis un an. Je m'adresse droit à vous comme au chef et au capitaine de cette troupe fameuse, qui s'est mise en profession de diffamer par ses injures tous ceux qui contredisent vos nouvelles maximes, et qui n'adorent pas vos sentiments. Je ne suis pas d'humeur à mordre la pierre que le Port-Royal a lancé contre moi, je vais droit au bras qui l'a jeté pour punir en son chef, la violence et l'injustice de ses membres. [...] / [...] Je sais de certitude que vous êtes l'auteur du titre injurieux du libelle, qui paraît contre moi sous le nom emprunté de Sainte Anne. Mais quand ce trait qui me blesse, serait parti de la main de l'un de vos soldats, ou que vous voulussiez dénier que vous en êtes l'auteur, je dois m'adresser à vous, comme au capitaine de cette troupe injurieuse, pour m'en faire raison. Car vous ne combattez qu'en troupes comme les Iroquois. [...] Et quand même quelqu'un des vôtres, pour vous mettre à couvert, se dirait à l'avenir l'auteur du libelle, et de l'ouvrage, qui m'a été fait avec tant d'injustice, je proteste hautement que je n'irai point à lui, qu'après que j'aurai fait avec vous ${ }^{74}$.» Pour Marandé, il n'est pas nécessaire que l'auteur des écrits à répondre soit le destinataire de ses réponses. Même si un écrit à réfuter provenait de la main de quelqu'un d'autre qu'Arnauld, Marandé écrirait toujours à celui-ci. Car Marandé qui compare Port-Royal à une armée parfaitement commandée par Arnauld, considère la réponse adressée à celui-ci plus efficace. C'est ainsi que la plupart de ses écrits polémiques viseront Arnauld.

En même temps qu'il écrit à Arnauld, Marandé essaie d'en extraire la réponse. A la fin de l'« avis à $\mathrm{M}^{\mathrm{r}}$ Arnauld», il écrit: "Au reste je ne prendrai point le change, et quelque adresse que vous puissiez employer pour m'engager dans une autre querelle, que celle dont il s'agit entre vous, je ne m'y attacherai point, que vous n'ayez auparavant satisfait sur tous les points que j'oppose contre votre doctrine, soit dans ce livre, soit dans les Inconvénients du Jansénisme que je vous adresse. Que si vous ne le faites pas, je réputerai votre silence, pour une conviction évidente de votre part, et pour un aveu nécessaire de tous les vôtres, des vérités de l'Église, que je soutiens contre vos nouvelles maximes ${ }^{75}$." En contraste avec l'attitude de l'auteur des Saints Pères de l'Église qui a essayé d'éviter la polémique avec Marandé, ce dernier exige des réponses d'Arnauld. Sa tentative de l'engager dans la polémique incarnera son attitude fondamentale de polémiste.

\section{Profiter du « jansénisme »?}

61 Dans les débats qui suivent les Antiquités de l'église, nous pouvons constater plus concrètement ces deux attitudes opposées. 
62 En ce qui concerne celle de Marandé, nous pouvons prendre comme exemple sa Réponse à la seconde lettre publiée en 1655. Marandé avait écrit la même année la réponse à la Première lettre d'Arnauld ; mais celui-ci n'y a pas répondu et même dans sa Seconde lettre il n'a fait que mentionner le livre des Inconvénients d'État sans citer le nom de Marandé. Suite au silence d'Arnauld, Marandé écrit dans sa Réponse à la Seconde lettre : «Je sais bien que le mépris qui semble enveloppé dans son silence affecté, je veux dire la suppression qu'il a faite de ma Réponse, aurait pu servir de motif suffisant à quelques autres, pour le piquer de nouveau, et le rendre plus sensible à la seconde qu'à la première attaque ${ }^{76} »$; «Et certes c'est en ce lieu, que l'on peut justement rapporter ce que cet écrivain dit dans sa Lettre par manière de plainte, quand il s'écrie que ses paroles nous offensent, et que son silence nous offense encore; puisque s'il parle de moi, c'est pour m'offenser par ses paroles injurieuses, comme il fait dans le milieu de sa Seconde Lettre; et s'il garde le silence à l'égard de ma Réponse, c'est pour m'outrager davantage par son mépris ${ }^{77}$ ». Le silence d'Arnauld a offensé Marandé aussi bien que ses paroles. Mais il me semble que pour Marandé qui cherche la polémique, les paroles d'Arnauld adressées à lui-même pouvaient aussi être une signe d'honneur, même si elles étaient injurieuses ; car il écrit dans la même Réponse: "Quoiqu'il en soit, je fais gloire du mépris de cet humble Docteur, et me tient fort honoré, de ce que si pour la défense des vérités de la foi, quelques-uns d'entre nos Doctes, participent aujourd'hui aux invectives de sa plume, je partage avec eux cet honneur dans quelque sorte de préciput, puisque j'ai part non seulement aux outrages de son silence, mais encore aux invectives de sa plume ${ }^{78}$. " S'il pouvait tirer gloire même des invectives, c'est qu'elles lui permettaient de prendre rang parmi les autres adversaires auxquels Arnauld écrivait. Autrement dit, pour Marandé, obtenir la réponse d'Arnauld était le moyen d'être reconnu comme polémiste.

Naturellement cette attitude de Marandé inspirait de l'aversion aux amis de Port-Royal. Une lettre d'Antoine Arnauld le montre clairement; cette lettre a été envoyée en 1661 à son frère Henri Arnauld quand Marandé a publié un pamphlet: Réflexions sur une lettre manuscrite pour attaquer la lettre de l'évêque d'Angers au roi. Arnauld écrit : « Il est bon que M. d'Angers soit averti qu'on a fait un libelle contre sa lettre, et qu'il sache quel est Marandé, auteur de ce libelle. C'est un écrivailleur sans jugement, qui a été autrefois greffier de la cour des aides, et qui s'est voulu ériger en théologien en traduisant quelque chose de la Somme de Saint Thomas. Rien ne satisferait tant sa vanité que de voir qu'on eût voulu prendre la peine de lui répondre ; mais c'est ce que l'on n'a point fait encore, et qu'on est résolu de ne point faire à l'avenir. Ignotus pereas miser necesse est. Il fut un de ceux qui écrivit contre la première lettre sur le sujet de l'affaire de M. Liancourt. Mais quoique dans la seconde on eût entrepris de répondre à neuf ou dix écrivains, on ne voulut pas seulement le nommer, de quoi il a pensé se désespérer. C'est pourquoi, si des personnes de la cour parlent de cet écrit à $\mathrm{M}$. d'Angers, il doit répondre [...] qu'il se gardera bien de s'arrêter à un petit écrivain qui prend pour fondement de son écrit une aussi grande impertinence qu'est celle de vouloir faire croire que la lettre d'un évêque présenté au roi par un ministre d'État est une lettre supposée; qu'un homme qui est capable de cette folie ne mérite d'être considéré que comme un fou et un impudent indigne de toute réponse ${ }^{79}$. " Ainsi dans les deux écrits pour la défense de l'évêque d'Angers, Arnauld n'a pas mentionné le nom de Marandé, et dans la Seconde lettre, tout en évoquant le titre des Inconvénients d'État, il n'avait pas cité le nom de l'auteur. De fait, malgré ses nombreux écrits polémiques, aucune réponse n'était adressée à Marandé. Cette attitude d'Arnauld est celle que nous avons vue dans l'auteur des Saints Pères de 
l'Église, et il est vrai que nous pouvons noter dans ces paroles le mépris pour le théologien amateur $^{80}$.

Cependant la critique d'Arnauld renfermait un autre élément, celui de la réputation. Car il écrira en 1689 dans sa Morale pratique des Jésuites : «On ne peut pas dire que c'est par impuissance qu'on ne répond point, quand on ne se dispense de répondre, qu'en se conformant au jugement du public. Or c'est ce qui arrive, quand on dédaigne de réfuter de petits Auteurs, qui, pour se faire un nom, s'avisent de prendre parti dans les querelles des savants; ou d'autres écrivains qui ne manquent pas d'esprit, mais qui se sont rendus eux-mêmes indignes de toute créance par leurs médisances et leurs calomnies. C'est pour cette raison que dans le même temps que l'on se donnait la peine de répondre sérieusement aux Pères Annat et Ferrier, on laissait aboyer les Marandés et les du Bosc, sans leur faire l'honneur de penser à eux ; [... $]^{81} »$. Arnauld choisit ses adversaires : il considère le père Annat, Ferrier et sans doute Claude Morel ${ }^{82}$, comme dignes de réponse, alors qu'il ne tient aucun compte des "petits écrivains» comme Marandé et Jacques Du Bosc, Cordelier ${ }^{83}$, parce que, d'après Arnauld, à la différence de ceux-là qui sont confesseurs du roi ou prédicateur du roi ${ }^{84}$, ayant de grandes connaissances en théologie, ceux-ci ne font que vouloir profiter des « querelles des savants " pour acquérir une certaine réputation. Arnauld a ainsi mis en question la poursuite de la gloire mondaine au moyen du débat théologique ${ }^{85}$.

\section{Conclusion}

En fin de compte, l'aversion que Port-Royal a eue à l'égard de Marandé se fondait sur un reproche, dont le point de vue n'a rien à voir avec la discussion théologique. Car les amis de Port-Royal reprochaient à un amateur en théologie, autrefois greffier de la cour des aides, de s'être mêlé de la querelle théologique afin d'acquérir une réputation. Certes, nous ignorons si la polémique de Marandé peut être réduite au problème de la gloire, comme l'a affirmé Arnauld. Mais en tenant compte de l'attitude de Marandé pendant la polémique ainsi que de ses deux parties de sa vie, il faudrait reconnaître que cette critique s'est révélée exacte jusqu'à un certain degré. À ce moment-là, les reproches provenant de Port-Royal nous permettent de comprendre plus concrètement la vie de Marandé que nous avons déjà suivie, parce que l'on peut y ajouter un nouvel élément, qui est la recherche de la réputation comme polémiste et écrivain, en profitant de l'affaire du "jansénisme». Nous avons déjà vu l'utilisation de la plume de Marandé dans le mouvement contre Port-Royal, mais, du point de vue de la réputation, on peut dire, à l'inverse, que c'est ce mouvement lui-même qui était l'objet de l'utilisation de Marandé.

On pourrait supposer en plus que les attitudes réciproques de Port-Royal et de Marandé révèlent une tendance plus générale, c'est-à-dire que la querelle janséniste du XVII ${ }^{e}$ siècle qui a eu un grand retentissement offrait aux "petits auteurs » comme Marandé et Du Bosc, l'occasion et le moyen d'essayer de se faire connaître dans le monde. Sans doute les paroles suivantes de Gabriel Gerberon se rattachent à cette tendance répandue jusqu'au greffe de la cour des aides : en expliquant la situation de l'année 1653 dans son Histoire générale du Jansénisme, il écrit : « Il est à remarquer que depuis que la bulle qui condamnait les cinq propositions fut reçue et publiée en France; les Jansénistes, je veux dire les théologien qui soutenaient la doctrine qui est vraiment celle de Jansénius et de Saint Augustin, s'y soumirent avec tant de respect, qu'ils ne publièrent aucun écrit, jusqu'à l'assemblée de quelques évêques, qui se tint l'année suivante au Louvre, mais pendant 
qu'ils se tenaient dans un si grand silence; les molinistes remplissaient le monde [de] leurs livres. L'on vit paraître cette année en France, outre ce que nous avons déjà marqué, la Pénitence publique d'un illustre janséniste : par un certain Léonard Marandé, greffier de la cour des aides, qui faisait le théologien, et était parfaitement ignorant, au moins en la doctrine de la grâce. Il nous donna aussi les Inconvénients du jansénisme adressés à $M$. Arnauld $^{86}$. » Aussi dans la description de l'année 1654, il écrit : « Il n'y eut pas jusqu'à un greffier de la cour des aides de Paris, qui ne se voulut signaler en écrivant contre le Jansénisme: le sieur de Marandé nous donna Les Inconvénients d'État procédant du Jansénisme ${ }^{87}$.»

67 Concluons. Suite à ses nombreuses attaques contre Port-Royal, Marandé était sans doute plus ou moins connu à cette époque parmi les gens ayant pris part au débat. Comme polémiste ou écrivain anti-janséniste, nous avons déjà vu qu'il a été présenté au roi par Jean Ferrier, ce qui n'aurait pas été possible dans le greffe de la cour des aides. Mais après sa mort, il tombe dans l'oubli, enseveli dans une foule d'ennemis mineurs de Port-Royal ; et aujourd'hui, si on peut lire son nom, c'est principalement grâce aux amis de Port-Royal qui étaient l'objet de ses attaques. Cependant en tenant compte des points de vue et des arguments de sa critique ainsi que de sa position et de son rôle dans le mouvement contre Port-Royal, il me semble que cet anti-janséniste oublié peut être l'objet d'une étude intéressante.

\section{NOTES}

1. Archives départementales du Cher, G94, $\mathrm{n}^{\circ}$. 29-34; E386; E840; E2417. Bibliothèque nationale, Pièces originales 1833 ; Mélanges de Colbert 120 bis, 121, 131 bis, 132.

2. Abraham Tessereau, Histoire chronologique de la grande chancellerie de France. Tome premier, contenant son origine, l'état de ses officiers, un recueil exact de leurs noms depuis le commencement de la Monarchie jusqu'à présent, leurs fonctions, privilèges, prérogatives, droits et règlements. Ensemble l'établissement et les Règlements des chancelleries près les cours de parlement, autres cours et sièges présidiaux du royaume. Le tout tiré des chartes, édits, déclarations, arrêts, règlements, registres et autres actes authentiques, par Abraham Tessereau écuyer, conseiller secrétaire du roi, maison, couronne de France et de ses finances. Revue et augmentée de plusieurs pièces, 1710, P. Emery, pp.626-627. La première édition ( 2 vol, t. 1 publié en 1676 chez P. Petit et t. 2 en 1706 chez P. Emery) ne contient pas les informations sur la famille.

3. Dans les années 1650, Mazarin l'a chargé au moins de deux missions : l'une est l'escorte du comte de Penaranda, plénipotentiaire espagnol aux négociations du traité de Westphalie, au cours de son retour en Espagne, et l'autre la négociation avec le comte de Daugnon, frondeur. «J'eusse été ravi de pouvoir parler à M. de Marandé ; car il est instruit à fond de l'affaire dont il me devait entretenir, j'eusse pu, en un quart d'heure, régler avec lui toutes choses, et lui dire franchement jusqu'où je pouvais aller » (Lettre à Servien du 18 octobre 1652, dans Lettres du Cardinal Mazarin, éd. Pierre-Adolphe Chéruel, 9 vol. , 1872-1906., t. 5, p. 406.). « [...] j'estime M. de Marandé fort homme d'honneur et que je me fie entièrement en lui [...] » (Lettre à Servien du 22 décembre 1652, Ibid, p. 512.). 
4. Henri Courteault, «Comment M. de Penaranda, premier plénipotentiaire d'Espagne à Munster, traversa la France en pleine Fronde» dans l'Annuaire-Bulletin de la Société de l'Histoire de France, 1924.

5. Épître dédicatoire mise en tête de l'édition anglaise: "To the right honourable, and truly noble, Edward Earle of Dorset, Lord Lieutenant of his Majesties Counties of Sussex, and Middlesex: Lord Chamberlaine to the Queene: One of the Lords of his Majesties most honourable Prior Councell, and knight of the most illustrious Order of the Garter » dans The judgment of Humane Actions a most learned, et excellent treatise of morall philosophie, which fights agaynst vanitie, et conduceth to the fyndinge out of true and perfect felicytie. Written in french by Monsieur Leonard Marrande and Englished by John Reynolds, London, A. Mathewes for Nicholas Bourne, 1629. Il est évident que le traducteur a en tête les Essais de Montaigne et De incertitudine et vanitate scientiarum et artium d'Agrippa.

6. Cf., René Pintard, Libertinage érudit, Slatkine, 1983 ( $1^{\text {re }}$ éd. 1943), p. $69 \mathrm{n}^{\circ} 1$, et p. 588. C'est $^{\prime}$ Charles Chesneau qui a écrit pour la première fois: "Cet ouvrage anonyme doit être de Marandé " (Le père Yves de Paris et son temps, t. 2, 1946, p. 407, $\mathrm{n}^{\circ} 79$. ), bien que dans sa bibliographie, il le classe dans les œuvres de Philippe de la Très Sainte Trinité. Nous considérons Marandé comme en étant l'auteur, selon l'opinion de Chesneau, mais nous reconnaissons quand même que le problème de l'auteur de cet ouvrage est à prouver avec plus de rigueur.

7. Le philosophe chrétien, ou les mystères de la foi, prouvés par raisons naturelles, F. Targa, 1639, p. 1. Le titre de la section première du Philosophe chrétien est «La raison naturelle s'accommode facilement avec les plus hauts mystères de la Foi ». Sur le terme "philosophe chrétien », voir une note importante d'Antony Mckenna: De Pascal à Voltaire. Le rôle des "Pensées" de Pascal dans l'histoire des idées entre 1670 et 1734, Oxford, The Voltaire Foundation, 1990, t. 1, p. 261, nº 41.

8. Après avoir présenté ces personnes, Saint Ange écrit : «Je pensais qu'il n’y eut que ces quatre qui en eussent parlé en notre langue, au mois n'en avais-je vu que ces quatre imprimés jusques à ces derniers jours que m'étant retiré du bruit de Paris à Montretou proche $\mathrm{S}$. Clou, où la courtoisie de mon Ami m'avait invité au contentement d'une agréable solitude, une personne d'esprit me fit présent du Philosophe Chrétien de Monsieur Marandé, l'état qu'il m'en fit, me donna la curiosité de le lire, les belles choses qu'il contient jointes à des sentiments extrêmement chrétiens, feront juger à tous ceux qui le liront que je ne pouvais lui refuser rang entre les précédents auteurs " (Le sieur de Saint Ange, La conduite du jugement naturel. Seconde partie, P. Blaise, 1641, pp. 65-66).

9. Les paroles suivantes peuvent révéler une certaine réticence de Marandé: «Si je donne d'abord quelque crédit à la raison, ce n'est qu'en qualité de servante de la foi, qui de vérité parle un peu haut en l'absence de sa Maîtresse, mais qui n'en abusera pas. Elle saura bien se taire en sa présence, et garder l'honneur et le respect qu'elle lui doit. Je sais bien que je lui fais prendre un vol au-delà de sa portée, entre ses mains ; mais sache qu'elle ne s'élève, qu'autant que ses forces lui peuvent permettre. » (Le philosophe chrétien, « avertissement au lecteur »).

10. Il est vrai que cette gratification est obtenue grâce à l'intervention de Pierre Scarron, évêque de Grenoble, par l'intermédiaire duquel Marandé a présenté son ouvrage à l'assemblée générale du clergé. L'assemblée était peu favorable à l'ouvrage de Marandé, à cause de son appréhension sur la vulgarisation de la théologie. De fait, ce n'était pas par l'examen de son œuvre que Marandé a reçu la prime, parce que dans l'assemblée, il a été arrêté «d'un commun consentement, que sans examiner l'ouvrage du Sr. Marandé, on lui donnerait 200 écus de gratification, par la seule considération de Mgr.de Grenoble » (Collection des procès-verbaux des assemblées générales du clergé de France, depuis l'année 1560, jusqu'à présent, t. 3, 1769, p. 98.). D'ailleurs on écrit dans le procès-verbal de l'assemblée : « Il fut dit qu'il fallait plutôt supprimer le livre que récompenser l'Auteur, et empêcher que la Théologie ne fût exposée en langue vulgaire, à raison de la faiblesse de plusieurs esprits, peu capable de digérer les mystères de la Religion » (Ibid., $\left.\mathrm{n}^{\circ} 1\right)$. 
11. Le Théologien Français, tome premier. Dans lequel selon l'ordre de l'école est traité des principes et propriétés de la Théologie, des attributs, de la vision, science, prédestination et volonté de Dieu, de la Trinité, des anges, et des lois. Par le Sr de Marandé, M. Soly, 1641, "Avertissement ».

12. Il y a une analyse important de Jean Mesnard sur la qualité littéraire du langage de cet ouvrage: "Langage littéraire et philosophie au XVII ${ }^{\mathrm{e}}$ siècle " dans Le langage littéraire au XVII siècle : de la rhétorique à la littérature, éd. Christian Wentzlaff-Eggebert, 1991.

13. Il y a une ressemblance frappante entre cet ouvrage de Marandé et celui de Saint Ange : il s'agit des deux parties de l'Abrégé curieux ("Logique » et " métaphysique ») et quelques sections de l'ouvrage de Saint Ange, qui sont également présentées sous forme du recueil des explications des mots ( $1^{\text {re }}$ partie de 1637 , section 2 : «Réflexion sur les quatre pas du raisonnement » et section 3: "Préparation à la direction du discours»; $2^{\text {e }}$ partie de 1641, $1^{\text {re }}$ partie: «Métaphysique, ou commencement des réflexions sur l'Etre »). La plupart des mots commentés dans les deux parties de Marandé sont les mêmes que ceux qui étaient présentés dans l'ouvrage de Saint Ange, et ils sont donnés dans le même ordre et de la même manière de l'explication que dans le livre de Saint Ange.

14. "Notre bien aimé le sieur de Marandé, nous a fait remontrer que dans le divertissement de ses Etudes plus sérieuses, il a composé un livre, auquel il n'a pas pu donner un titre plus convenable que Le Théologien français » ( le privilège du roi donné au Théologien français, daté du 16 juin 1640) ; «Ce petit ouvrage m'a servi de divertissement et de relâche en mon grand travail » (L'esprit de Grenade sur La Guide des Pécheurs [...], corrigé et augmenté en cette nouvelle édition, T. Blaise

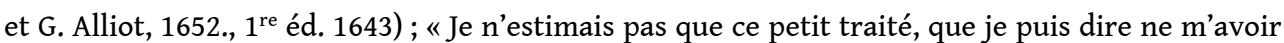
coûté de temps, qu'autant qu'il en faudrait pour le transcrire, dût être si bien reçu... » (Abrégé curieux et familier de toute la philosophie, logique, morale, physique et métaphysique, et des matières plus importantes du Théologien français, $4^{\mathrm{e}}$ éd., M. Soly, 1645., "Au lecteur »).

15. La clef ou abrégé de la Somme de S. Thomas, divisé en deux tomes, M. Soly, 2 vol., , 1649, t. 1, "Raisons du motif et de l'ordre de cet ouvrage».

16. Hilarion de Coste, La vie du R.P. Marin Mersenne, S. et G. Cramoisy, 1649, p. 83.

17. Adrien Baillet, La vie de Monsieur Descartes, D. Hortemels, 2 vol. , 1691, t. 2, p. 146.

18. Leonardi Marandaei Ariades, C. Cramoisy, 1629, épître mis en tête : "Illustrissimis et sapientissimis venetiarum principibus. Leonardus Marandaeus, S. D. ».

19. Jean de Warignies, seigneur de Blainville (vers 1581-1628).

20. On trouve à la Bibliothèque nationale une lettre datée du 26 novembre 1627, qui est adressée à Marandé par Blainville. Elle évoque la première œuvre de Marandé, ou ses pensées montaigniennes, car en se plaignant « de ne voir que fort rarement dans le lieu de ma solitude les choses qui m'en peuvent plaire, et d'y recevoir à la foule les traverses et les ennuis ", Blainville écrit: «Ce n'est pas que j'aie l'esprit assez faible pour les tenir tel que je les nomme, car vous m'avez appris par une conversation d'en connaître la différence et de ne donner le titre de bien ou de mal qu'à ce qui l'est en vérité ; c'est ce qui a fait parmi la presse la pompe que traîne d'ordre un ambassadeur après soi. Je me suis souvent préparé contre le lustre d'une si fausse vanité, de peur que si mes sens abusés ne pussent en trahissant mon âme la porter à des espérances trompeuses. Cette prémédité disposition m'a fait recevoir sans douleur la privation de toutes ces choses " (Collection Dupuy 5 , fol. $129 \mathrm{r}^{\circ}$ ).

21. Richard H. Popkin, Histoire du scepticisme, traduit par Christine Hivet, P.U.F., 1995 (éd. anglaise, 1979), p. 133 et 144.

22. Adrien Baillet, Ibid., t. 2, p.146. «Il semble qu'on pourrait aussi rapporter au temps de la demeure de M. Descartes à Paris, l'amitié qu'il eut avec M. Frenicle, qu'il appelle souvent M. de Beffy simplement; avec M. de Sainte Croix, M. de Marandé, et M. Picot, quoi que je n'aie pu encore fixer le commencement de leur connaissance ».

23. Hilarion de Coste, op.cit., p. 83. 
24. Lettre de Mersenne à André Rivet, datée du 10 novembre 1640, dans Correspondance du $P$. Marin Mersenne, éd. Cornélis de Waard, Édition du CNRS, 17 vol. , 1932-1983., t. 10, pp. 219-220. Voir aussi, Marin Mersenne, La vérité des sciences contre les sceptiques ou pyrrhoniens, 1625, éd. Dominique Descotes, Champion, 2003, Livre I, chapitre 16, et l'« Introduction ", notamment pp. 30-31.

25. Marin Mersenne, Harmonie universelle, contenant la théorie et la pratique de la musique, S. Cramoisy, 1636, Première préface générale au lecteur, $10^{\mathrm{e}}$ page non numérotée.

26. Camus écrit à Marandé : «Et quoi que dans la fameuse question des aides de la grâce, vous teniez une opinion qui n'est pas tout à fait celle à qui je donne les mains (comme je vous ai autrefois fait entendre en conversation privée) si est-ce que je n'improuve pas la vôtre, la tenant fort plausible » (Lettre de J. P. Camus à Marandé, datée du 14 février 1641. Cette lettre est jointe au premier volume du Théologien français.).

27. En ce qui concerne l'éloge qu'a fait Camus sur les ouvrages de Marandé, voir la lettre du 14 février 1641 ainsi qu'une autre lettre de Camus à Marandé, qui se trouve attachée en tête du premier volume de La clef de St Thomas sur toute sa Somme (1668). Cependant celle-ci porte sans doute sur les Morales chrétiennes du Théologien français.

28. «Etat général du payement que le roi veut et ordonne être fait à ses officiers domestiques, par son conseiller et trésorier général de sa Maison, [...], à commencer du premier jour de janvier 1650 et finissant jusques à ce que sa Majesté en ait fait autre nouvel état », dans l'État général des officiers domestiques et commensaux de la Maison du Roy, de la Reine, \& de Monsieur le Duc d'Anjou, qui doivent jouir des privilèges, M. Le Ché, 1652, p. 5. Voir aussi l'État général des officiers domestiques et commensaux de la Maison du Roy, M. Le Ché, 1657, p. 3.

29. Le privilège du roi donné le 31 juillet 1651 à son ouvrage intitulé les Antiquités de l'église (1652).

30. Le Théologien français, [...]. Troisième édition revue, corrigée et augmentée par l'auteur, t. 1, M. Soly, 1652, « avis au lecteur ».

31. Guillaume de Peyrat, L'histoire ecclésiastique de la cour; ou les antiquités et recherches de la chapelle, et oratoire du roi de France, depuis Clovis I. Jusques à notre temps, H. Sara, 1645, p. 468.

32. Adrien Baillet, Ibid., t. 1, pp. 146-147.

33. Nous pouvons donner comme témoins Godefroi Hermant (ses Mémoires, éd. Augustin Gazier, 1905-1910, t. 1, pp. 590-591. et t. 5, p. 489), Sieur de Sainte Anne (Les Saints Pères de l'Église vengés par eux-mêmes, 1652, p. 4), Antoine Arnauld (sa lettre de 1661 adressée à l'évêque d'Angers, dans les Mémoires d'Hermant, t.5, p. 406.), Abbé de S. Eleuthère (Remarques de Monsieur l'Abbé de S. Eleuthère, sur une Lettre que le sieur Marandé, ci-devant Commis au greffe de la cour des aides de Paris, a écrite à un R. P. Capucin de Languedoc, s.l.n.d., p. 6. Cet écrit est au plus tôt de l'année 1664), Gabriel Gerberon (Histoire générale du Jansénisme, t. 3, 1701, p. 278) et Louis-Ellies Du Pin (Histoire ecclésiastique du dix-septième siècle, t. 2, 1714, p. 363). En plus on peut constater que la première édition du Théologien français (1641-1643) est certainement publiée par le "greffier » Marandé alors que la quatrième édition (1664) l'est par le « conseiller et aumônier du roi » Marandé.

34. Adrien BAILLET (1649-1706). Il était le dirigé de G. Hermant. Il a écrit La vie de Godefroi Hermant (Amsterdam, 1717).

35. Antiquités de l'Église, «Préface de la conduite de Dieu sur l'Église, et des usages différents qui sont survenus dans sa discipline» (sans numérotation). Nous présentons aussi les phrases qui précèdent cette citation : "Il m'est arrivé à peu près, comme à celui qui sort de son pays dans un temps de paix, pour faire un long voyage, et qui à son retour, trouve la guerre civile et le feu de la division entre ses concitoyens. Car m'étant engagé dans un long voyage, je veux dire dans le grand ouvrage de la Théologie, dans un temps calme et paisible entre les Savants, je m'étais réservé de traiter des cérémonies des Sacrements, et de leurs anciens usages, après que j'aurais accompli cette longue course; de crainte qu'une matière se féconde, étant traitée en son lieu naturel, et à la fin de chaque Sacrement, ne cause quelque disproportion difforme dans le corps 
de l'Ouvrage. Mais étant retourné sur mes pas pour achever mon dessein, j'ai trouvé que l'inconstance des choses humaines, qui n'a pas moins de part dans les sentiments, que dans les mœurs, avait causé quelque tumulte et quelque légère division sur la pratique de quelques Sacrements, dont l'usage est le plus fréquent et le plus ordinaire dans l'Église. Cette rencontre imprévue me surprit d'abord, et la crainte que j'avais qu'on ne se persuada que je voulusse entreprendre de dire mon avis sur une matière si délicate, qui a servi d'emploi à tant de plume disertes et éloquentes, me retint quelque temps en suspens, pour consulter en moi-même, s'il ne serait pas plus à propos de manquer à ma parole, que de poursuivre le dessein que je m'étais proposé dès le commencement de mon ouvrage. Mais [...]» (Ibid.).

36. D'autres titres par exemples : Traité 7, Discours 1 : «Que selon l'usage qui s'est pratiqué dans l'Église depuis Saint Cyprien jusques à Saint Augustin, tous les pécheurs publics n'étaient pas toujours exclus de la communion des fidèles, ni de la participation des saints mystères "; Traité 14, Discours 1: «De la satisfaction et de sa nécessité, pour les peines temporelles qui sont dues de notre part, après la rémission de la coulpe et de la peine éternelle, selon la doctrine des Pères ». Cette thèse est celle qui sera critiquée dans la $10^{\mathrm{e}}$ Provinciale dans laquelle Pascal remet en question l'adoucissement du sacrement de la pénitence dans la morale jésuite.

37. Les paroles suivantes de Marandé nous rappellent aussi la $5^{\mathrm{e}}$ Provinciale qui traite de deux sortes de casuistes, relâchés et sévères, qu'ont les jésuites pour contenter tout le monde. " [...] comme l'effet d'une justice sévère et rigoureuse, devait devancer en la personne de Jésus-Christ, le fruit de sa miséricorde [...], aussi était-il convenable que l'Église qui est le corps mystique de Jésus-Christ, imita son chef autant qu'il était possible, et qu'elle marqua dans son enfantement, les traces des souffrances de son chef, avant que de goûter les consolations de son fruit et de sa lignée, [...]. Ce n'est pas que l'Église ne doive par ses larmes pénitentes et continuelles, achever en ses membres, les souffrances qui manquent ou qui défaillent à la Passion de Jésus-Christ, comme parle l'Apôtre ; ce n'est pas qu'il nous suffise de révérer les rigueurs de l'ancienne Pénitence, qui a fleuri durant quelques siècles dans l'Église, et de les respecter, comme les anciens titres de notre noblesse, que nous conservons dans nos Archives, sans y toucher sous peine de faux ; qu'au contraire nous les devons renouveler, non pas tant par des actes publics [...] que par des actes intérieurs de charité, d'obéissance, de mortification, d'humiliation et d'anéantissement, et par des actes extérieurs de l'aumône de l'assistance des malades, [...]. / Quand donc nous disons que la douceur, a succédé à la sévérité ; nous ne prétendons pas que la sévérité, ne soit maintenant nécessaire dans le régime des âmes, aussi bien que la douceur ; [...] Après tout, la douceur et la sévérité sont toujours nécessaires dans la conduite des âmes ; l'une et l'autre est vertu ; [...] aussi est-il absolument nécessaire, que ceux qui sont commis au régime des âmes, dont les unes ont besoin de sévérité, et les autres de douceur, prennent garde de ne pas rendre l'une trop molle et trop lâche, et l'autre trop austère et trop dure ; car si la trop grande douceur, est parfois capable de causer dans les âmes quelques négligence mortelle dans les choses du salut, il est à craindre pareillement, qu'une trop grande sévérité ne se rende insupportable, et qu'elle n'enfante le désespoir dans les âmes timides, qui est le plus grand de tous les maux. Mais il faut selon la différence des personnes et de leurs infirmités, que la prudence chrétienne fasse emploi de l'une ou de l'autre, pour la guérison des plaies spirituelles ; et que parfois elle les mélange, pour former le divin médicament de l'Evangile, composé d'huile et de vin » (Antiquités de l'Église, «Préface de la conduite de Dieu... ", sans numérotation).

38. Godefroi Hermant, op.cit., t. 1, pp. 590-591.

39. Inconvénients d'Etat procédant du jansénisme avec la réfutation du Mars français de Monsieur Jansénius, S. et G. Cramoisy, 1654, p. 97.

40. Sur ce problème, il y a un article de Christian Nadeau: "Conscience nationale et raison d'État. Le Mars gallicus de Jansénius et la critique de Léonard de Marandé ", dans Port-Royal et l'Histoire, Chroniques de Port-Royal, nº 46, 1997. 
41. Inconvénients d'État, « Au roi », sans numérotation. Il continue : «Ce fera pour lors que votre Majesté (selon saint Augustin), évitant le blâme qu'ont encouru tous les rois, qui du temps des Prophètes n'ont point interdit par leur loi, ni renversé par la puissance de leur sceptre, les choses qui s'introduisaient furtivement dans leurs peuples contre les ordres de Dieu, elle acquerra les titres magnifiques, et les plus sublimes éloges des Princes, dont les vertus et les mérites ont été élevés au-dessus de tous les autres Souverains, pour avoir détruit et renversé les Idoles de l'erreur, qui prétendaient s'établir sous votre Règne dans le peuple chrétien, contre l'honneur et le culte véritable de Dieu ».

42. Blaise Pascal, Les Provinciales, éd. Cognet et Ferreyrolles, 1992, p. 290.

43. L'étude importante sur l'histoire de cette lettre circulaire est l'article de Lucien Ceyssens: «La lettre circulaire de Port-Royal: un faux indestructible», dans Jansenistica Minora, t. 12. Article publié pour la première fois en 1973 dans Bulletin de l'Institut historique belge de Rome, $\mathrm{n}^{\circ}$. 43.

44. Constitution de N. S. P. le Pape Innocent X. par laquelle sont déclarées et définies cinq propositions en matière de foi, A. Vitré, 1655, pp. 55-57.

45. Dans le recueil Constitution de N. S. P. le Pape..., «il est arrivé que le dernier décret de sa Sainteté, du 29 septembre 1654, dans lequel nous lisons la condamnation [...] a été traduit par quelqu'un qui favorise ce nouveau parti, ainsi qu'il est à présumer par les falsifications remarquables, par la visible corruption des principaux points qu'il contient, et par l'omission affectée de quelques termes efficaces çà et là diversement répandus dans ce Bref ; mêmes de quatre ou cinq paroles consécutives en quelques endroits, pour énerver dans l'esprit des ignorants, la force de ce Décret. / Cet abus, dont la grandeur et la conséquence ne peuvent être suffisamment exprimées par des termes communs et ordinaires, étant parvenu à la connaissance des principaux Ministres de la Justice :[...] pour obvier aux désordres qui pourraient naitre de cette insigne corruption, et empêcher que sous ce prétexte les Novateurs ne puissent tromper les peuples, et les induire en erreur sur cette matière, contestée depuis tant de temps, et enfin si glorieusement décidée, pour la paix et le repos de l'Église; il a été commandé au sieur de Marandé Conseiller et Aumônier du Roi, de traduire ce même Décret, d'en conférer ensuite avec les Docteurs, afin qu'en sa traduction il ne s'y trouve rien à redire; qu'un chacun par ce moyen puisse apprendre dans la sincère version de ce Bref, l'intention de sa Sainteté, avec la doctrine et le sens véritable des choses qu'il contient» («Avis sur la nouvelle impression du dernier Décret d'Innocent X. en date du 29 Septembre. 1654.» dans la Traduction fidèle..., p. 3-4.). Cependant nous ne savons pas exactement la situation curieuse autour de cette traduction favorable à Port-Royal, car celle-ci est publiée avec permission chez Antoine Vitré, «imprimeur ordinaire du roi et du clergé de France ", et est probablement l'une des pièces envoyées à tous les prélats suivant la décision de l'assemblée particulières de 15 prélats tenue le 20 mai 1655 sous la présidence du cardinal Mazarin.

46. Relation des délibérations du clergé de France, sur la constitution, et sur le bref de N.S.P. le Pape Innocent X. par laquelle sont déclarées et définies cinq propositions en matières de foi, A. Vitré, 1656, pp. 71-73. Touts les points de corrections que Marandé a fait remarquer dans sa traduction sont adoptés. Voir aussi la Collection des procès-verbaux des assemblées générales du clergé de France, t. 4 , 1770, pp. 29-68.

47. Réflexion sur la censure, S. et G. Cramoisy, 1656, p. 5.

48. «Un libelle de Port-Royal » est la Défense de la Constitution du Pape Innocent X. et de la Foi de l'Église; contre deux livres dont l'un a pour titre, Cavilli Jansenianorum, ... et l'autre Réponse à quelques demandes... (1655, attribué à Lalane ou à Arnauld). Quant à la « retraite des docteurs jansénistes ", il s'agit de l'affaire qui a eu lieu dans l'assemblée de Sorbonne du 24 janvier 1656 pour la censure d'Arnauld: soixante docteurs favorables à lui sont sortis de cette assemblée en manifestant leur opposition contre les mesures qui diminuent et limitent le temps attribué à chacun pour parler. La « protestation » d'Arnauld désigne l'écrit latin qu'il a présenté à la Faculté 
de théologie le 27 janvier 1656 juste avant la décision de la censure concernant la question de droit, afin de protester contre les fausses procédures de la censure (Voir Euvres de M. Antoine Arnauld, t. 19.).

49. La première tentative de réponse est intitulée : Lettre écrite à un abbé par un docteur sur le sujet des trois premières lettres écrites à un provincial, datée du 22 février 1656.

50. Considérations sur un libelle de Port-Royal..., S. et G. Cramoisy, 1656, « Au lecteur ».

51. Ibid., pp. 113-114.

52. Les écrits mis en questions par Marandé sont les trois premières parties de l'Éclaircissement du fait et du sens de Jansénius etc. contre les livres, écrits et extraits de MM. Pereyret, Morel, Chamillard, Annat, Amelotte et autres (166l, attribué à Lalane selon Hermant ou à Claude Girard selon Quesnel), ainsi que De l'Hérésie et du Schisme causé par l'exaction de la signature pure et simple du Formulaire (1661, attribué à Arnauld et Nicole).

53. Réflexions sur une lettre manuscrite qui paraît sous le nom de Monseigneur l'Évêque d'Angers, s.l.n.d. [1661], p. 3. La lettre de l'évêque d'Angers, en manuscrit au moment de la réponse par Marandé, sera publiée: Lettre de Monseigneur l'évêque d'Angers au roi pour la signature du formulaire du clergé, s.l.n.d..

54. L'un est un texte assez court : l'« Avis sur un autre Libelle, contre la Lettre de Monseigneur l'Évêque d'Angers au Roi » qui est attaché à la fin de l'œuvre pour la défense du "premier mandement ", intitulée : Défense de l'Ordonnance de Messieurs les Vicaires Généraux de Monseigneur le Cardinal de Retz, Archevêque de Paris, pour la signature du Formulaire; l'autre est un écrit intitulé : Éclaircissement sur le différend entre Jean d'Antioche et S. Cyrille, dont il est parlé dans la Lettre de M. L'Évêque d'Angers au Roi.

55. Guillaume Le Roi, Lettre sur la constance et le courage qu'on doit avoir pour la vérité. Avec les sentiments de S. Bernard sur l'obéissance qu'on est obligé de rendre aux supérieurs, et sur le discernement qu'on doit faire de ce qu'ils commandent, tirés de sa VII Lettre, s. 1., 1661.

56. Lettre de Jean Ferrier à Marandé, datée du 5 juillet 1662, dans les Mémoires d'Hermant, t. 5, pp. 489-490.

57. Voir une lettre de Marandé écrite sans doute en automne 1664 et adressée à Simplicien, capucin de Languedoc, publiée dans les Remarques de Monsieur l'abbé de S. Eleuthère, sur une lettre que le sieur Marandé, ci-devant commis au greffe de la cour des aides de Paris, a écrite à un R. P. Capucin de Languedoc pour le consoler, de ce qu'en suite de quelques sermons qu'il avait faits dans la ville de Toulouse, il avait été interdit par les grands vicaires de l'archevêché, s.l.n.d.. La même lettre de Marandé se trouve également dans un écrit intitulé : L'interdit de deux RR. PP. Capucins dans la ville et le Diocèse de Toulouse (s.l.n.d.), pp. 4-5.

58. Selon Sainte Anne, auteur des Saints Pères de l'Église (1652), dans le livre des Antiquités de l'Église Marandé « entreprend de parler de la satisfaction, touchant laquelle il pose deux maximes qu'il prétend de prouver. La première, que dans l'ancienne église l'absolution sacramental, se donnait toujours immédiatement après la confession des péchés, quels qu'ils fussent, ou publics, ou secrets, et avant l'exercice des œuvres satisfactoires. La seconde, que cette manière de procéder, c'est-à-dire, de donner toujours l'absolution immédiatement après la confession, était nécessaire" (Les saints Pères de l'Église, p. 3.). Sur la seconde proposition, Sainte Anne écrit que Marandé a puisé ses sources dans le livre de Brisacier de 1651 : Jansénisme confondu dans l'avocat du sieur Callaghan, car il écrit : «Il [Marandé] est encore en quelque façon pardonnable, parce qu'il n'est pas seul dans cette erreur. Il a pour compagnon un grand personnage, et des célèbres de notre siècle, le $\mathrm{P}$. Brisacier jésuite, dans ce grand et savant ouvrage qu'il publia sur la fin de l'année dernière [1651], [...] Et il y a grande apparence que c'est de là que le sieur de Marandé l'a pris, puisqu'il cite le même lieu du sage, et se sert du même raisonnement, et que c'était en ce temps-là qu'il composait son livre, l'ayant fait imprimer au commencement de cette année [1652], [...]. Il est bien vrai que s'il n'eût pas tant ajouté de foi sur la parole et l'autorité de ce guerrier et martial jésuite, il ne se serait peut-être pas tant hâté, et s'il ne se fût pas tant hâté, il 
aurait trouvé l'antidote de ce venin dans le livre qui a paru depuis peu contre celui de ce jésuite, sous le titre de l'Innocence et la vérité défendue, etc. » (Ibid., pp. 51-52.).

59. Hermant, Mémoires, t. 5, p. 489.

60. Ibid., p. 490.

61. Arnauld écrit : «il [Ferrier] présenta au roi le sieur Marandé, et lui recommanda l'auteur et son livre, qui est l'ouvrage le plus injurieux et le plus envenimé qu'on se puisse imaginer et qui ne respire que le feu et le sang et qui ne parle que d'exterminer et de perdre tous ceux qui refusent de signer le formulaire " (Lettre à Arnauld d'Andilly, datée du 6 mai 1663, dans CEuvres de M. Antoine Arnauld, t. 1, pp. 363-364.).

62. François Annat, Réponse à quelques demandes dont l'éclaircissement est nécessaire au temps présent. Seconde Édition augmentée des réflexions sur la seconde lettre du sieur Arnauld, F. Lambert, 1656 (1 ${ }^{\mathrm{re}}$ éd. 1655).

63. Réflexion sur la censure (décembre 1655) et Réponse à l'écrit que Monsieur Arnauld a fait présenter (février 1656).

64. Arnauld écrit : «Ce libelle (qui n'est qu'un extrait des Inconvénients d'État du sieur Marandé) a deux parties. La première n'est qu'un amas d'impertinences, de faussetés et d'impostures contre M. Jansénius, et M. de S. Cyran. La seconde, qui commence à la page 12, est ce qui en fait le capital, et auquel le reste n'a servi que de préambule ; car on voit assez que votre but n'a été que d'empoisonner les simples par les mensonges de la prétendue Lettre circulaire de Messieurs de Port-Royal. Mais pour les faire mieux recevoir, vous avez usé de deux ou trois supercheries. 1) En mettant un avis du sieur Marandé sur cette Lettre, vous n'en avez laissé que les douze premières lignes, et vous en avez retranché tout ce qui faisait voir qu'il n'en parlait qu'en doutant, et qu'il déclarait qu'on la devait regarder comme une pièce supposée, si ceux dont elle portait le nom la désavouaient. 2) Vous avez mis au bas du frontispice de cet extrait : À Paris, chez Sébastien Cramoisi 1654, avec privilège. Cela devrait marquer en la place où il est mis, que cet extrait a été imprimé à Paris chez Cramoisi ; ce qui est une fausseté pour l'autoriser davantage. Car c'est le gros livre de Marandé qui a été imprimé à Paris par Cramoisi, à son grand dommage, en 1654, au lieu que l'extrait n'a été imprimé qu'aux Pays-Bas, plus de douze ans depuis; et vous auriez eu honte de le faire voir à Paris. [...]» (Antoine Arnauld, Morale pratique des jésuites, tome VIII des précédentes éditions. De la calomnie ou instruction du procès entre les jésuites et leurs adversaires, sur la matière de la calomnie, 1695, dans Euvres, t. 35, pp. 121-122.).

65. Oorsprong en oorsaeken der Jansenisterye, Menin, 1675, in-24, 32 p. Cf., Léopold Willaert, Bibliotheca Janseniana Belgica, J. Vrin, t. 1, p. 1949, p. 282.

66. Léopold Willaert, op.cit., t. 1, p. 284. Nous n'avons pas encore consulté cet extrait, mais ce titre nous rappelle quelques chapitres des Inconvénients d'État: Article 5 : «Que la providence de Dieu sur la France, l'avait prémunie d'un saint préservatif contre les erreurs du Jansénisme », Section 2: «Que la doctrine du B. François de Salles Évêques de Genève, devait servir de bouclier aux Fidèles, contre les nouvelles erreurs de l'Évêque d'Ypres »; Section 3: "Parallèle de quelques maximes extraites du livre de Monsieur Jansénius, intitulé l'Augustin d'Ypres, avec la doctrine orthodoxe du B. François de Sales Évêque de Genève ».

67. Journal historique et littéraire, Maestricht et Liège : F. Cavelier et J.F. Bassompierre, t. 3, 1791, septembre, pp. 13-22.

68. Ibid., p. 4.

69. François Annat, La conduite de l'Église et du roi, justifiée dans la condamnation de l'hérésie des jansénistes. Par la réfutation des faux prétextes de la question de fait et de droit: et de la prétendue conformité de leur doctrine avec celle des Thomistes : et par la preuve de leur véritable conformité avec les calvinistes, S. et M. Cramoisy, 1664. Denis Amelote, La Défense des constitutions d'Innocent X et d'Alexandre VII et des décrets de l'Assemblée générale du Clergé de France, contre la doctrine de Jansénius contenue aux cinq propositions condamnées, S. Huré, 1660. Cf., Léopold Willaert, op.cit., t.2, pp. 307-308.

Courrier du Centre international Blaise Pascal, 26 | 2015 
70. Godefroi Hermant, Mémoires, t. 5, p. 489. et p. 491.

71. D'après Hermant, l'auteur est Jacques Brousse, docteur de la maison de Navarre. Selon le Dictionnaire de Moréri (éd. 1759), cet écrit est attribué à Étienne de Lombard, abbé du Trouillas ou à Toussaint-Gui-Joseph Desmares, oratorien. Cioranescu l'attribue à Desmares ou à Lalane.

72. Claude Morel ( ?-1679), docteur de Sorbonne, prédicateur du roi en 1640, doyen de la faculté de théologie. Peut-être collaborateur de la rédaction des cinq propositions. Il a fait de la polémique avec Arnauld.

73. Le Sieur de Sainte Anne, Les saints Pères de l'Église vengés par eux-mêmes des impostures du sieur de Marandé dans son livre des Antiquités de l'Église, s.l., 1652, p. 4. L'auteur continue à expliquer sa raison : «Il est vrai qu'à son défaut, il semble que je me devais adresser à Messieurs Guichard et Sauffoy, Docteurs et Professeurs en Théologie de votre Faculté, qui ont honoré ce livre de leur approbation avec éloge : leur remontrer le peu de soin qu'ils apportent dans l'examen des livres ; et leur reprocher l'injure, et la honte, qu'ils font à un corps aussi célèbre, qu'est celui de Sorbonne, de le rendre protecteur d'un ouvrage si monstrueux. Mais deux choses m'ont retenu. L'une qu'étant professeurs, ils pourraient prendre prétexte de leur emploi, pour se dispenser avec quelque sorte de couleur de me faire réponse. L'autre, qu'étant encore jeunes docteurs, et s'étant attachés à cet exercice de la scolastique dès le temps qu'ils n'étaient encore que bacheliers et avant qu'ils eussent lu les Pères : c'est leur faire honneur en cette occasion, et courir une partie de leur honte, de dire d'eux ce que j'ai dit du sieur de Marandé ; que l'obligation de leurs leçons les a divertis de se pouvoir instruire dans l'École de la tradition, autant qu'il serait nécessaire pour n'être point trompés, et ne tromper point dans l'examen des ouvrages de cette nature : et que la créance qu'ils ont avec beaucoup d'autres, que les jésuites sont infaillibles dans leur doctrine, aussi bien qu'impeccables dans leurs mœurs, leur fait souscrire à l'aveugle, et approuver avec éloge tout ce qui vient de leur part, ou de celle de leurs amis, et qu'ils savent certainement n'avoir travaillé que sur leur mémoire et sur leur correction. Ainsi, Monsieur, vous voyez que c'est avec raison que je ne m'adresse point au sieur de Marandé, ni à Messieurs Guichard et Sauffoy pour l'éclaircissement de ces difficultés, quoi qu'ils semblent y être les plus intéressés. Or si parmi le reste des savants de votre Société de Sorbonne, je m'adresse particulièrement à vous, deux raisons m'y obligent lesquelles comme je crois vous ne contredirez point, parce qu'elles vous sont honorables. La première est votre qualité prétendue de Censeur Royal et extraordinaire. Car cette qualité vous établissant le docteur des docteurs, et le censeur des censeurs; lors qu'il est question du privilège pour l'impression d'un livre, l'on n'a point d'égard au sceau et à l'approbation des autres docteurs, si elle n'est autorisée du contrôle et de l'attache de la vôtre: de sorte qu'encore que votre nom, ne paraisse point dans cette approbation, ni dans ce privilège, non plus que dans ceux des autres livres qui s'impriment, il ne faut pas douter que vous ne l'ayez vu et approuvé, Messieurs Guichard et Sauffoy étant encore trop jeunes, et trop peu connus dans le Conseil, pour y obtenir sous leur simple attestation, ce que l'on a refusé sous celle de beaucoup d'autres, plus anciens, plus capables, et plus connus, à moins que d'avoir celle de Monsieur Morel. La seconde raison qui me fait avoir recours à vous, est, qu'étant vieux docteur comme vous êtes, l'un des plus célèbres et des plus versés à ce que disent vos amis, dans la lecture de la Tradition, qui soit en toute la maison de Sorbonne : qui par un long et fameux exercice en la prédication de l'Évangile, avez acquis avec tant de mérite la noble et glorieuse qualité de conseiller et prédicateur du roi, et qui d'ailleurs n'êtes point employé dans une lecture publique. Je me suis facilement persuadé que vous auriez assez de charité pour donner quelques heures de votre loisir, pour l'éclaircissement d'une matière aussi importante qu'est celle-ci. Joint qu'ayant vue déjà quelques-uns de vos ouvrages, où vous avez réussi si heureusement et avec tant d'adresse, pour faire ce que nul autre que vous n'aurait pu faire, c'est-à-dire passer, la confession de Pélage, ce grand ami de la grâce de Jésus-Christ, comme vous savez pour la confession de foi de S. Augustin : il y a grande apparence que vous aurez quelque secret que les autres n'ont pas, pour justifier les erreurs du sieur de Marandé, et faire 
voir qu'il a bien entendu les Pères, lors qu'il les a cités contre leur esprit, contre leur dessein, et contre leurs propres paroles ; ce qui vous a obligé de trouver bon qu'on donnât un privilège à son livre afin qu'il fut débité avec plus de pompe et plus de solennité » (Ibid., pp. 4-6.).

74. Pénitence publique d'un illustre janséniste, adressée à M. Arnauld, S. et G. Cramoisy, 1653, "Avis à $\mathrm{M}^{\mathrm{r}}$ Arnauld » (sans numérotation).

75. Ibid.

76. Réponse à la seconde lettre de $M^{r}$ Arnauld, S. et G. Cramoisy, 1655, p. 2.

77. Ibid., pp. 2-3.

78. Ibid., p. 2.

79. On trouve cette lettre sans date, bien qu'incomplète, dans les Mémoires d'Hermant, t. 5, pp. 406-407.

80. Nous voyons ce mépris plus nettement exprimé par un auteur qui a écrit au plus tôt en 1664 sous le nom de l'abbé de Saint Eleuthère les remarques ironiques sur Marandé : «Ce n'est pas tant l'occasion de servir l'église, que cherche le sieur Marandé, comme celle de paraître partout, de se mêler de toutes les disputes du temps, de régner et de triompher parmi des semi doctes, et des semi théologiens »; " ce que l'Église pourrait désirer de lui s'il en était capable ; ce serait qu'il eut à se taire, et laisser juger des questions de théologie à Messieurs les prélats, et à la Sorbonne : car pour ne lui rien celer, on se lasse de voir un petit clerc de greffe, qui n'a pas la moindre teinture des belle lettres, qui ignore les principes de la philosophie, qui n'a aucune connaissance de l'histoire ecclésiastique, ni des sentiments des SS.PP. et qui avec cela n'a nul caractère, ni mission, devenu docteur en commençant d'être écolier, se mêler à tort et à travers de dogmatiser, et d'écrire des mystères inconcevables de notre religion, et particulièrement de ceux de la grâce, où il ne voit goutte" (Remarques de Monsieur l'abbé de S. Eleuthère, sur une lettre que le sieur Marandé, s.l.n.d., p. 2.). Mais nous savons peu de choses sur cet auteur.

81. Antoine Arnauld, Morale pratique des jésuites, troisième volume des précédentes éditions, contenant la justification des deux premiers volumes de cette Morale, contre le livre faussement intitulé: Défense des nouveaux chrétiens et des missionnaires de la Chine, du Japon et des Indes, 1689, dans CEuvres, t. 33, p. 125.

82. Arnauld a répondu à Morel. Contre les Véritables sentiments de Saint Augustin (1650), il a écrit : Apologie pour les saints Pères de l'Église, défenseurs de la grâce de Jésus-Christ. Contre Les jansénistes convaincus d'erreur et de mensonge (1657), il a écrit une partie de l'Éclaircissement du fait et du sens de Jansénius. Il a répondu aussi à La conduite de saint Augustin contre les pélagiens (1658).

83. Il a laissé de nombreux écrits contre Port-Royal. Sur sa vie et son œuvre, voir l'article d'Antoine de Sérent: "Histoire littéraire et bibliographique. Jacques du Bosc ", dans La France Franciscaine. Mélanges d'archéologie, d'histoire et de littérature, Lille, 1912, pp. 219-231. Voir aussi un autre article d'A. Sérent : «Les frères mineurs en face du jansénisme (1607-1754) », dans Études franciscaines, nouvelle série $n^{\circ} 5,1951$, pp. 213-228. Marandé avait le point de vue politique en considérant le «jansénisme » comme secte d'État, secte politique, tandis que chez Du Bosc nous trouvons le point de vue philosophique, car il écrit lui-même de son premier ouvrage contre Port-Royal: «Le Philosophe indifférent, en l'année 1643, qui traite de l'origine des sectes. Je l'ai ainsi nommé, parce que j'oppose l'indifférence et la liberté en raisonnant, à l'affectation des sophistes. Je montre en ce livre comme les sectes des novateurs sont nées des sectes des philosophes, ceux qui ont déchiré les vérités révélées, étant comme les enfants et les disciples de ceux qui ont déchiré les vérités naturelles: [...]. Ayant dessein de combattre l'hérésie, je fis d'abord cet ouvrage qui est comme un fondement, et s'il faut ainsi dire, une place d'armes pour mon dessein. » (Jacques Du Bosc, «Le catalogue des livres que l'auteur a donnés au public sur la controverse de notre siècle ", cité dans les deux articles d'A. Sérent.).

84. La raison pour laquelle Sainte Anne s'est adressé à Claude Morel se fonde sur la qualité théologique de ce prédicateur du roi. Voir les paroles de Sainte Anne dans la note 73. 
85. Nous ajoutons encore deux petites citations : la première est celle d'Hermant; il parle de Marandé dans la description de l'année 1661 : «comme il aspirait depuis longtemps à l'honneur d'obtenir une réponse de $\mathrm{M}$. Arnauld ou de quelqu'un de ses amis, il multipliait ses écrits pour en venir à ses fins » (t. 5, p. 406.) La seconde est celle de Saint Eleuthère dont on a déjà mentionné : «Il y a longtemps que tout le Jansénisme serait exterminé, s'il avait voulu : mais il ne veut pas ruiner entièrement cette Carthage, pour avoir de quoi exercer sa valeur ». Il me semble que ces paroles suggèrent l'utilisation de la querelle janséniste qui sera mise en cause dans la critique d'Arnauld.

86. [Gabriel Gerberon], Histoire générale du Jansénisme, contenant ce qui s'est passé en France, en Espagne, en Italie, dans les Pays-Bas etc. au sujet du Livre, intitulé, Augustinus Cornelii Jansenii, Amsterdam : J. Louis de Lorme, t. 3, 1701, p. 278.

87. Ibid., t. 3, p. 391.

\section{INDEX}

Mots-clés : Marandé (Léonard de), jansénisme, anti-jansénisme, Port-Royal

Keywords : Jansenism, anti-Jansenist

\section{AUTEUR}

\section{KEISUKE MISONO}

CERHAC, Université Blaise Pascal

doctorant 